\title{
POD and Fourier analyses of a fluid-structure-acoustic interaction problem related to interior car noise
}

\author{
Éric Gaudard ${ }^{1,2}$, Philippe Druault ${ }^{2, a}$, Régis Marchiano ${ }^{2}$ and François Van Herpe ${ }^{1}$ \\ 1 PSA Peugeot Citrỏen, Research Department, Centre Téchnique de Vélizy A, Route de Gisy, 78140 Vélizy-Villacoublay, France \\ 2 Sorbonne Universités, UPMC Univ Paris 06, CNRS, UMR 7190, Institut Jean Le Rond d'Alembert, 75005 Paris, France
}

Received 11 September 2015, Accepted 29 March 2016

\begin{abstract}
In order to approach a flow configuration revealing the aerodynamic noise contribution in the interior of road vehicles due to the A-pillar vortex, a numerical simulation of a Forward Facing Step (FFS) coupled with a vibrating structure is performed. This numerical study is based on a weak coupling of three solvers to compute (i) the flow field in interaction with the FFS, (ii) the vibration of the structure and (iii) the acoustic radiation in the open cavity. The purpose of this work is then to evaluate the ability of two different post-processing methods: Proper Orthogonal Decomposition and Fourier Decomposition to identify the origin of the noise radiated into a cavity surrounded by an unsteady flow. Fourier and POD decompositions are then successively performed to extract the part of the aeroacoustic wall pressure field impacting the upper part of an upward step mainly related to the radiated acoustic pressure in the cavity. It is observed that the acoustic part, extracted from the wavenumber frequency decomposition (Fourier analysis) of the wall pressure field generates a non-negligible part of the interior cavity noise. However, this contribution is of several orders smaller than the one related to the aerodynamic part of the pressure field. Moreover, it is shown that the most energetic part of the pressure field (POD analysis) is due to the shear flapping motion and mainly contributes to the low-frequency noise in the cavity. Such post-processing results are of particular interest for future analyzes related to the noise radiated inside a car.
\end{abstract}

Key words: Aeroacoustic analysis / proper orthogonal decomposition / Fourier analysis / interior car noise

\section{Introduction}

The development of quieter car engines and of hybrid road vehicles has led to consider aerodynamic noise as an important source of interior noise especially at mid and high driving speeds. The aerodynamic noise comes primarily from the vortex shedding at the A-Pillar location which interacts with the Turbulent Boundary Layer (TBL) developing on panel windows. This vortex shedding on the window creates wall pressure excitations that cause noise inside the vehicle, leading to some discomfort for the driver and passengers. In order to reduce the interior noise limiting the driver's fatigue, the level of noise induced by structural vibrations in the cavity has to be predicted. Then the development of post-processing tools aimed at characterizing the flow-structural interaction is essential to better understand and predict the interior acoustic field. Such analysis performed in a realistic flow configuration remains today quite difficult. We then propose to test the ability of two post-processing tools

\footnotetext{
${ }^{a}$ Corresponding author: philippe.druault@upmc.fr
}

applied to a simplified flow-structure-acoustic interaction problem which approaches the A-pillar vortex flow configuration. A numerical study of the acoustic radiation in an open cavity induced by a vibrating structure forced by an unsteady flow is then considered in this paper. It is assumed that there is no source of sound in the open cavity and that the only mechanism of sound production is given by the coupling between the cavity and the vibrating structure. The purpose of this work is to study the aeroacoustic part of the wall pressure field which is responsible for the acoustic noise in the cavity. In this sense, we do not want to generate additional acoustic modes associated with the cavity properties that justify the choice of an open cavity. The vibrations of the structure are then only due to the unsteady pressure acting on its surface: the wall pressure. The wall pressure can be viewed as the superposition of two components: (i) the pressure due to acoustics waves propagating in the flow, (ii) and the unsteady aerodynamic pressure flow. A simple configuration to observe this situation is to consider the flow around a forward facing step (FFS) and to consider that the elastic structure is located behind the FFS (see Fig. 1 


\section{Nomenclature}

\begin{tabular}{|c|c|}
\hline CFD & Computational Fluid Dynamic \\
\hline DNS & Direct Numerical Simulation \\
\hline FT & Fourier Transform \\
\hline FFS & Forward Facing Step \\
\hline POD & Proper Orthogonal Decomposition \\
\hline PSD & Power Spectral Density \\
\hline RANS & Reynolds Averaged Navier-Stokes \\
\hline RMS & Root Mean Square \\
\hline TVD & Total Variation Diminishing \\
\hline$E$ & Young modulus of the structure \\
\hline$a^{(n)}(t)$ & $n$th temporal POD coefficient \\
\hline$c$ & Phase velocity, \\
\hline$e$ & Thickness of the structure \\
\hline$f$ & Frequency \\
\hline$f_{e}=\frac{1}{\Delta t}$ & Sampling frequency \\
\hline$I$ & Second moment of inertia of the structure \\
\hline$k_{0}$ & Acoustic wavenumber \\
\hline$k_{c}$ & Turbulent convection wavenumber \\
\hline$k_{s}$ & Structural wavenumber \\
\hline$k_{x}$ & Wavenumber along the $x$ direction \\
\hline$\left(L_{x}, L_{y}\right)$ & $\begin{array}{l}\text { Physical dimension of the FFS } \\
\text { flow domain }\end{array}$ \\
\hline$\left(L_{x}^{\prime}, L_{y}^{\prime}\right)$ & Physical dimension of the cavity domain \\
\hline$n_{t}$ & Number of instants \\
\hline$\left(n_{x}, n_{y}\right)$ & $\begin{array}{l}\text { Number of grid points of the FFS } \\
\text { flow configuration }\end{array}$ \\
\hline$\left(n_{x}^{\prime}, n_{y}^{\prime}\right)$ & Number of grid points of the cavity domain \\
\hline$p(x, y, t)$ & Pressure signal at position $(x, y)$ and time $t$ \\
\hline$U_{\infty}$ & Uniform streamwise flow velocity \\
\hline$s$ & Entropy, \\
\hline $\mathbf{X}=(x, y)$ & Two-dimensional space variables \\
\hline$x_{v i b}$ & $\begin{array}{l}x \text {-coordinate of the one dimensional } \\
\text { vibrating structure, }\end{array}$ \\
\hline$Y$ & Transverse displacement of the structure \\
\hline$\hat{p}\left(k_{x}, f\right)$ & Spatio-temporal FT of the pressure signal \\
\hline$\Delta t$ & Time step, \\
\hline$(\Delta x, \Delta y)$ & Mesh-grid discretization along $(x, y)$ directions \\
\hline $\begin{array}{l}\Delta f \\
\Phi^{(n)}(x)\end{array}$ & $\begin{array}{l}\text { Frequency resolution } \\
n \text {th spatial POD eigenfunction }\end{array}$ \\
\hline$\rho_{s}$ & Density of the structure \\
\hline$\rho$ & Density of the fluid \\
\hline$\tau$ & Viscous stress tensor \\
\hline
\end{tabular}

for a schematic representation). In practice, this configuration occurs in presence of detached flows impinging an elastic structure and when the sound field behind the structure matters. For instance, this configuration can be viewed as a very simplified reproduction of the aerodynamic noise contribution in the interior of a road vehicle due to the A-pillar, even though the simulation is here two-dimensional. Nevertheless, this study does not claim to reproduce the real $3 \mathrm{D}$ configuration but is an attempt to link aerodynamic events to acoustic radiation in a cavity for a two-dimensional configuration.
The study of aerodynamic noise generated from flowexcited structures involves the investigation of the wall pressure fluctuations on the structure, the structural vibrations excited by the surface pressure field, and finally the sound radiated from the vibration of the structure. The complete investigation of such flow-structure coupling and of the resulting sound radiation is quite complex and time consuming. Only few authors have already performed such an analysis on a simplified flow configuration $[1,2]$. A full study needs to take into account the complex exterior flow field (interaction of A-pillar vortex, TBL flow, door mirror) that generates unsteady wall pressure fields impacting the vibrating panel window and leading to interior noise. Apart from the difficulties in numerically investigating such a complex flow, simplified flow configurations are generally retained allowing the investigation of particular flow events responsible for interior noise in the cabin. For instance, a lot of previous studies have proposed to model the structural loading analytically based on deterministic or random excitations [3-6]. In this case, the structural loading usually includes random, distributed excitations, such as diffused acoustic fields and aerodynamic excitations. Then, the structural vibrations and acoustic radiations are computed from the analytical model representing wall pressure excitation [5].

The difficulty of such modeling concerns the restitution of the complex aeroacoustic wall pressure field excitation and its associated physical mechanisms. Indeed, this excitation is composed of a turbulent component and an acoustic one $[3,7,8]$ and both components contribute to the interior noise. Another solution may consist in performing a numerical simulation of similar flow configurations. Thus, to reproduce a flow configuration approaching the A-pillar vortex, a Forward Facing Step (FFS) configuration is considered. This simplified geometry allows the generation of an aeroacoustic wall pressure field impacting the upper part of the step corresponding to a vibrating structure.

Even though forward facing step has been intensively studied in the past in incompressible subsonic flows (see for instance [9-11] and references therein), the analysis of compressible FFS flow has been much less investigated. Based on previous experimental and numerical database, it has been shown that the main region containing acoustic sources is located in the recirculating flow close to the step corner. The far-field acoustic of FFS flow configuration has been experimentally investigated by several authors [12-15]. Recent acoustic measurements exhibit the dependence of the step height on the acoustic spectra [15]. Moreover, the acoustic source seems to be of dipolar nature [12-14]. Numerical simulations have also been performed for aeroacoustic analysis of FFS flow configuration [16-19]. For instance, based on incompressible Large Eddy Simulations (LES) of the FFS flow coupled with Lighthill's analogy formulation to access the far-field acoustic, Ji and Wang [16] showed that the front step acts primarily as a dipole source aligned in the streamwise direction. Previous studies have assumed that, for 


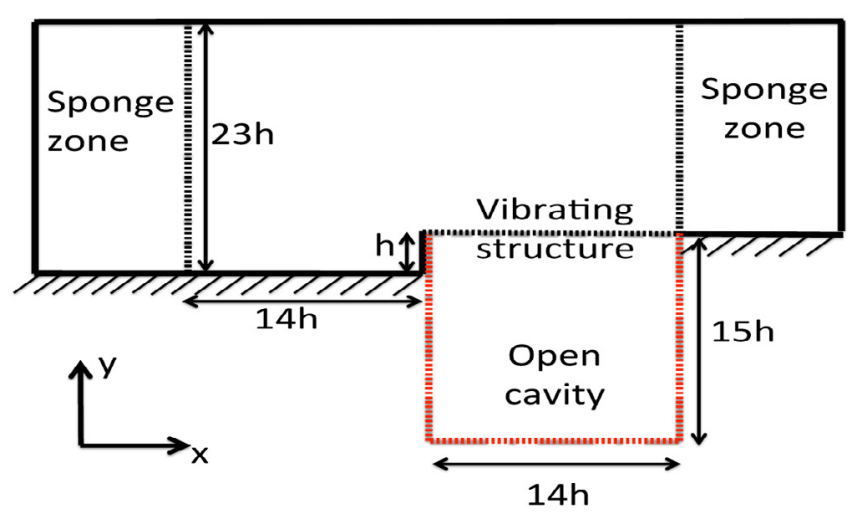

Fig. 1. Schematic representation of the three flow domains: exterior forward facing step, vibrating structure and open cavity (not to scale). The sponge regions of the open cavity domain and also at the upper transverse boundary domain are not represented for clarity.

low-Mach number $(M \approx 0.1)$ FFS configurations, the dominant acoustic contribution comes from the step front flow impact. However, very recent experimental and theoretical analyses $[20,21]$ demonstrated that the noise due to wall pressure fluctuations may dominate the other contributions for various Mach numbers FFS flow. Awasthi et al. [22] have also demonstrated that the wall pressure spectrum levels are related to the step height of the forward-facing step. Thus, the characterization of the wall pressure signal including not only acoustic but also hydrodynamic fluctuations, remains a great challenge to elucidate the flow mechanisms responsible for the structure vibration and then for the radiated noise in a cavity.

As the present study is a preliminary one focusing on the flow-structure-acoustic interaction, it needs to be simplified to better investigate the origin of the radiated sound in the cavity. It is then proposed to perform a direct noise computation of the two-dimensional (2D) FFS configuration. Note that the numerical simulation of similar fluid-structure-acoustic interaction in a $3 \mathrm{D}$ context leads to a very high computational cost. But, even if a 2D computation is not able to take into account the $3 \mathrm{D}$ flow structures developing in the recirculation area [23,24], it allows the description of the main flow characteristics. Moreover, Wilhelm et al. [23] performed a comparative analysis of 2D and 3D numerical simulation of FFS flow configurations and similar mean flow properties are recovered in both computations.

The objective for the present study is to identify the wall pressure contribution which is mainly related to the radiated acoustic pressure in a cavity. More precisely, we attempt to identify unsteady flow characteristics (shear layer flapping motion, eddy structure interacting with the wall, ... ) that could be mainly related to the noise radiated in an open cavity located under the step. For such an investigation, two common post-processing methods are tested: proper orthogonal decomposition (POD) and Fourier decomposition. These modal analyses are ones of the most popular flow decomposition techniques used for the analysis of the experimental and/or numerical space- time database. POD and Fourier analysis both allow a space-time description of the flow dynamics as a set of statistical quantities referred as eigenfunctions. For the POD technique, eigenfunctions are determined based on the energy content [25]. Conversely, for Fourier decomposition, the modes are related to the wavenumber and/or frequency content of the available database. These flow decompositions have demonstrated in the past their efficiency in identifying the flow structures present in turbulent flow. More precisely, POD technique allows the detection and the characterization of the large scale energetic coherent structures [25] while Fourier analysis extracts periodic flow structures. Note also that the Fourier technique is generally more devoted to noise and vibration analysis thanks to spectral analysis. In a previous paper, Gaudard et al. [26] have already analyzed the effectiveness of these two methods but in a different context. Indeed, in this last paper, a synthetic wall pressure field was used to test the effectiveness of both mathematical tools (POD and FFT) to discriminate the acoustic and aerodynamic parts of the synthesized aeroacoustic wall pressure field. In the present paper, we propose to test the ability of these two methods in a fluid-structure-acoustic interaction context related to a simplified flow configuration allowing to state about which aeroacoustic events that can be associated with the main noise contribution in the cavity.

In Section 2, the selected configuration and numerical solvers are presented. Section 3 is devoted to the analysis of the forward-facing step flow. The results of the decomposition of the wall pressure using two different processing tools are discussed in Section 4. The analysis of the radiation of the elastic structure is given in Section 5 .

\section{Numerical solvers and fluid-structure configuration}

A schematic illustration of the configuration and the computational domains is given in Figure 1. As already mentioned, the goal of this study is to find links between a pressure recorder in the open cavity and the wall pressure generated by the flow in the exterior domain on the vibrating structure. From a theoretical point of view, each physical domain can be associated with a particular model. In the exterior domain, as explained in the introduction, we would like to simulate the effect of the unsteady flow over the structure as well as the acoustic waves generated by the flow and radiated. Therefore, one has to deal with the compressible Navier-Stokes equations. The vibrating structure is a beam. Assuming small amplitudes of deformation it is reasonable to consider a linear and isotropic Euler/Bernoulli model. Finally, the open cavity is supposed to be at rest with no flow. Moreover, the propagation can be considered linear, hence the use of the classical wave equation to model that domain.

To predict the acoustic pressure field in the cavity, three successive computations are done: (1) a twodimensional Navier-Stokes solver is used in the exterior 
domain especially to compute the wall pressure, (2) a one-dimensional beam solver simulates the structural vibrations due to the unsteady wall pressure, (3) a twodimensional acoustic solver allows to access to the radiated acoustic field in the open cavity.

In the following parts these three solvers are briefly described and the associated numerical parameters used in simulations are provided.

\subsection{Navier-Stokes solver and numerical flow parameters}

The wall pressure is computed in the exterior domain from the Navier-Stokes equations:

$$
\begin{aligned}
\frac{\partial \rho}{\partial t}+\nabla \cdot(\rho \mathbf{v}) & =0 \\
\rho\left(\frac{\partial \mathbf{v}}{\partial t}+(\mathbf{v} \cdot \nabla) \mathbf{v}\right)+\nabla p & =\nabla \cdot \tau, \\
\frac{\partial s}{\partial t}+(\mathbf{v} \cdot \nabla) s & =0 .
\end{aligned}
$$

with $p(\mathbf{X}, t)$ and $\rho(\mathbf{X}, t)$ the pressure and density field depending on the space and time variables. $\mathbf{v}(\mathbf{X}, t)$ is the velocity vector and $s(\mathbf{X}, t)$ the entropy field. $\tau$ is the viscous stress tensor. The fluid is supposed to be newtonian.

These equations are solved with a 2D numerical solver called CAAMELEON and already described in the following References [27-29]. CAAMELEON solver allows the Direct Numerical Simulation of the compressible 2D Navier-Stokes equations. However, in the present test case, the grid resolution is not sufficient to ensure that all time-space flow scales of the flow are resolved. Thus, here, the grid discretization acts as a filtering procedure for the Navier-Stokes equation resolution. This code solves the pseudo-characteristic formulation of the Navier-Stokes equations that provides a decomposition of the pressure, velocity and entropy fluxes $[28,30]$. Equations are written in generalized coordinates and the code is able to solve any configurations on a curvilinear grid [30]. To enforce both numerical stability and accuracy, a fourth-order accurate upwind DRP scheme is used and this scheme is modified near the computational domain boundaries [27]. Time integration is performed using a third-order TVD Runge-Kutta scheme. The code is parallelized with a domain decomposition.

To reproduce previously published FFS flow configurations $[9,16]$, the step height is fixed to $h=0.0127 \mathrm{~m}$ and a uniform streamwise velocity field $U_{\infty}=25 \mathrm{~m} . \mathrm{s}^{-1}$ is imposed in the far field. The Reynolds number $R e_{h}=$ $U_{\infty} h / \nu$ is around 20000 . The physical computation domain extends from $-14 h$ to $14 h\left(L_{x}=28 h\right)$ in the streamwise direction and from $-h$ to $22 h\left(L_{y}=23 h\right)$ in the transverse direction (see Fig. 1). The computational meshgrid is not uniform in both directions. As mentioned before the code can deal with curvilinear grids. The transverse grid discretization is uniform $d y / h=1 / 300$ for $y / h \in[-1: 0.5]$ and then the transverse discretization is regularly stretched along that direction until reaching a maximum mesh size of $d y / h=1 / 10$. In a similar manner, the streamwise discretization is uniform $d x / h=1 / 100$ for $x / h \in[-1: 1]$ and it is regularly stretched in both directions $x / h<-1$ and $x / h>1$ with a maximum mesh step of $d x / h=1 / 10$. The total number of points for the physical domain under investigation is $\left(n_{x}, n_{y}\right)=(510,720)$ excluding the sponge regions. The CFL number is chosen equal to 0.5 based on the smallest mesh size. Considering this CFL number and the meshgrid, the time step of the simulation is equal to $5.947 \times 10^{-8} \mathrm{~s}$. The coordinate system origin is located at the upper corner of the step. To avoid any spurious reflexions, sponge regions are added along the streamwise direction (left and right-boundary) and also at the upper $y$-boundary. Sponge layers are made by applying a stretching on the grid in the direction of interest. The stretching is progressive to avoid any spurious reflection at the boundary between the physical domain and the sponge layer (the stretching coefficient is 0.028). The size of the sponge layers is chosen to be large enough to ensure the damping of the waves and of the aerodynamic structures before the computational boundary (168 points in the $x$-direction and 137 points in the $y$-direction corresponding approximately to $12.5 \%$ of the computational domain for each of them). Note that, in the following figures, flow variables will only be represented in the physical domain.

Such a flow configuration is very sensitive to initial conditions [23]. To initialize it, the initial state (set of pressure, density and velocities in the whole domain) is computed by solving the incompressible RANS equations with the open source CFD software OpenFoam [31,32]. It provides a statistically well converged mean flow field related to the FFS flow configuration. A uniform and cartesian mesh of $(1000 \times 750)$ points is used to discretize the $(x, y)$ domain of the FFS configuration. A laminar boundary layer is imposed in inflow. The model for turbulence is the classical $k-\epsilon$ model. This method allows us to save an important time comparatively with a naive initialization of the code with, for instance, a uniform state in the whole domain. Nevertheless, this procedure requires to interpolate the steady solution obtained by OpenFoam to the grid described above.

The initial conditions are now available and correspond to a uniform mean streamwise flow velocity of $U_{\infty}=25 \mathrm{~m} . \mathrm{s}^{-1}$ outside the mean Blasius velocity field obtained near the wall. Based on the pseudo-characteristic formulation of the Caameleon solver, it is not necessary to generate at each time step this streamwise velocity field at the left boundary domain [27]. Indeed, in such a formulation, only the flow derivatives have to be imposed at the left streamwise boundary of the computational domain. Thus, imposing a null derivative of the velocity derivative fluxes at this boundary is similar to generate a uniform velocity field.

Regarding the grid resolution associated with the Boundary Layer (BL) flow, the classical normalized values of $x^{+}$and $y^{+}$are respectively equal to 13.2 and to 4.4 based on the determination of the friction velocity when the TBL flow is developed. These values are obtained at 
$x / h \approx-3.5$. Such a grid resolution is sufficient to accurately predict the aeroacoustic flow variables.

More than $3.6 \times 10^{6}$ time steps have been computed (about $108 \mathrm{~h}$ on $64 \mathrm{CPUs}$ ). Once the initial condition is entirely evacuated, the flow variables are stored every 500 time steps, therefore the sampling frequency is $33630 \mathrm{~Hz}$. We consider that the data are significant after 2000 time steps. Indeed, beyond that number of time steps, the different statistical quantities studied in the next paragraphs are converged. Hence, the database containing the flow variables is made of 5000 samples for each point of the mesh grid. That leads to an analyzable time duration of $0.1488 \mathrm{~s}$ which corresponds approximately to ten times the time travel of a fluid particle advected through the complete flow domain at the uniform speed of the flow. Note that the time duration of the simulation may place some constraints on the following stochastic data analysis. However, when performing Fourier, the frequency domain covers $[0$ :16815] Hz with a frequency step of $6.7 \mathrm{~Hz}$. These values seem quite sufficient for the present investigation. Indeed, the flow structure passage is largely inferior to $10000 \mathrm{~Hz}$ and the frequency resolution provides a sufficient accuracy for the characterization of the present aerodynamic events.

The wall pressure is extracted from this database. The spatial coordinate of the vibrating structure will be referred to as to $x_{v i b}$ indicating that $x$ is varying from 0 to $14 h$ and $y=0$. Thus, the wall pressure denoted $p\left(x_{v i b}, t\right)$ is then used as a source term for the elastic solver.

\subsection{Euler-Bernoulli beam model solver and numerical parameters}

The model used here is based on the hypothesis that one-way interactions occur: first, the fluid outside the cavity engenders vibrations of the structure, then, the vibrations of the structure radiate acoustical waves in the cavity. We do not consider a full-coupling approach. Some preliminary tests based on a full-coupling approach in a very simplified configuration were performed during the Ph.D. thesis of Gaudard [33]. In this context, the error done for a non-full coupling approach was less than $1 \%$ compared to the full-coupling approach. In this study, it is expected that the full-coupling approach would not modify the present results. Indeed, the aeroacoustic wall pressure field is of very high energy content comparatively to the energy's level of the acoustic radiation in the cavity and then of the internal acoustic vibrations. Then, we assume that the one way coupling leads to modify only less than a few percents the solution comparatively to the solution of a full-coupling approach.

Moreover, the interaction between the flow and the structure is assumed to be small enough to consider only small deformations. It is also supposed that the structure is unaffected by the acoustic response inside the cavity. Only the transverse displacements (in the $y$-direction) of the structure $Y\left(x_{v i b}, t\right)$ are considered.

According to these assumptions, the vibrations are modeled with the one-dimensional Euler-Bernoulli equa- tion with supported ends. The fluid load excitation denoted $\Delta p\left(x_{v i b}, t\right)$ corresponds to the difference between the wall pressure field described above $\left(p\left(x_{v i b}, t\right)\right)$ and the uniform pressure field initially present in the open cavity:

$$
m \frac{\partial^{2} Y\left(x_{v i b}, t\right)}{\partial t^{2}}+E I \frac{\partial^{4} Y\left(x_{v i b}, t\right)}{\partial x^{4}}=\Delta p\left(x_{v i b}, t\right),
$$

where $E, I$ and $m$ are respectively the Young modulus, the second moment of inertia and the linear mass of the structure. This structure is supposed to be a glass with the following properties: thickness $e=0.001 \mathrm{~m}$, Young modulus $E=70 \times 10^{9} \mathrm{~Pa}$, density $\rho_{s}=2500 \mathrm{~kg} \cdot \mathrm{m}^{-3}$.

The transverse displacement is computed based on the finite element method. The structure is meshed with 139 equally spaced elements [33] and the time integration is done by using a Newmark's scheme [34] with a time step of $3 \times 10^{-5} \mathrm{~s}$.

\subsection{Acoustic wave propagation solver}

Knowing the transverse displacement of the structure, it is possible to compute the normal velocity of the structure and to use it as an imposed boundary condition to compute the acoustic field in the open cavity. To compute the acoustic field in the cavity, the previously described two-dimensional Navier-Stokes solver is used. The dimensions of the cavity located under the vibrating structure are $\left(L_{x}^{\prime}, L_{y}^{\prime}\right)=(14 h, 15 h)$ that corresponds to a uniform mesh in both directions of $\left(n_{x}^{\prime} \times n_{y}^{\prime}\right)=(140 \times 150)$ points with a constant space discretization of $d x^{\prime} / h=d y^{\prime} / h=$ $1 / 10$. The time step of this simulation is $2.478 \times 10^{-6} \mathrm{~s}$. The initial condition corresponds to a medium at rest with a uniform pressure field, $p_{0}=101325 \mathrm{~Pa}$. Instantaneous fluctuating velocity field computed based on the beam model solver is used as inflow condition (upper transverse boundary domain) at each time step of this numerical simulation. As the time step of the beam solver response is smaller than the time step of the Navier-Stokes solver, a linear interpolation of the transverse velocity field in time is done before its generation as inflow condition. In the other boundaries, a sponge region is imposed to avoid any spurious reflexions. More than 60000 time steps are simulated. Once the acoustic field is established in the cavity, 12000 instantaneous acoustic pressure fields are stored with a sampling frequency of $403560 \mathrm{~Hz}$. That corresponds to a time duration of $0.03 \mathrm{~s}$. We have in mind that any limited time duration places some constraints on data analysis, especially in the frequency resolution which is then only of $33.6 \mathrm{~Hz}$. However, for the present acoustic data analysis, this discretization seems to be sufficient to properly describe the nature of the acoustic signals in the cavity and also to perform a comparative analysis of the efficiency of flow decomposition methods. 

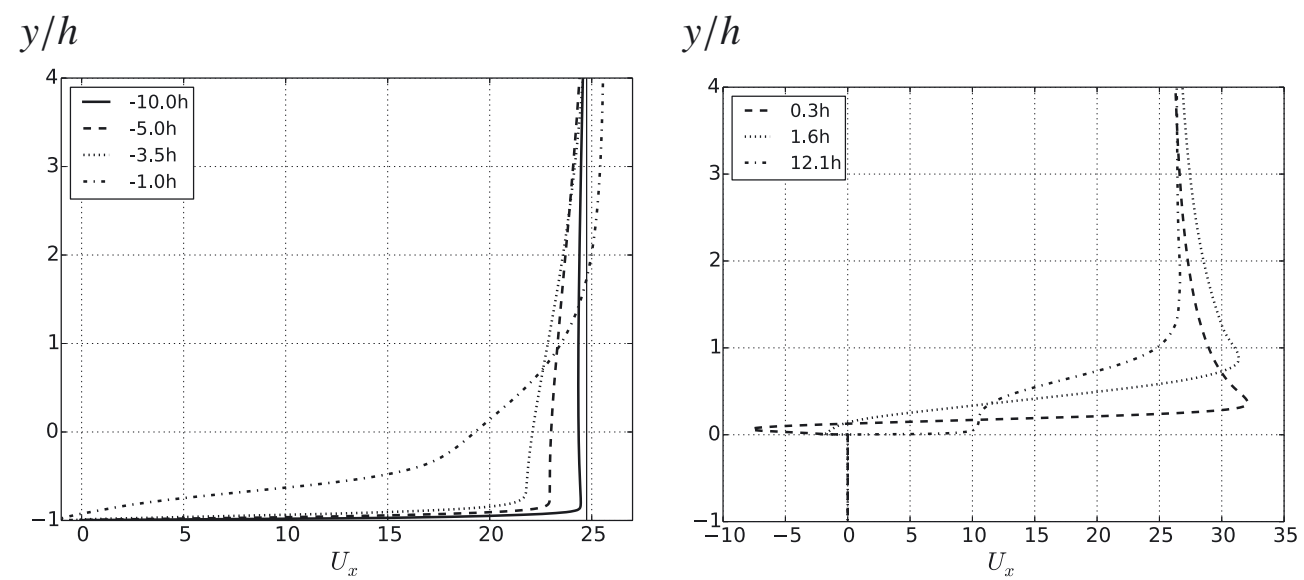

Fig. 2. Near wall profiles of mean streamwise velocity extracted at selected $x$-locations.

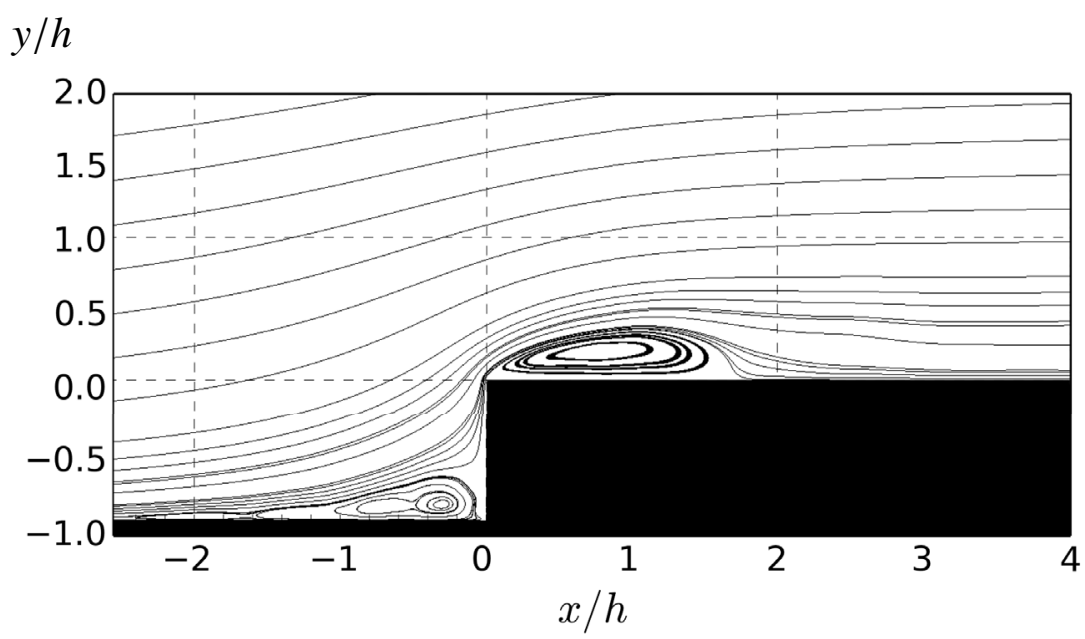

Fig. 3. Streamlines of the mean velocity field.

\section{Aeroacoustic analysis of forward-facing step flow}

\subsection{Mean flow properties}

The streamwise mean velocity profiles extracted at selected $x$-locations are presented in Figure 2. The thickness of the turbulent boundary layer developing before the flow separation is of an order of $2.4 h$. Two separation regions are retrieved in front of and on the step (see streamlines of the mean velocity field in Fig. 3). The recirculation region downstream of the step extends to $2 h$ which is in reasonable agreement with previous investigations [11,35]. Such recirculation area corresponds to the flapping motion of the shear layer. As expected the turbulent kinetic energy is higher in the upper recirculation region than in other regions due to the increase in the shear of the mean velocity field [33].

\subsection{Wall pressure analysis}

The pressure fluctuations obtained at the wall (before and after the step) denoted $p_{\text {wall }}$ are now investigated.
Figure 4 displays two representative wall pressure coefficients defined as follows:

$$
C_{p_{\text {wall }}}=\frac{p_{\text {wall }}-p_{0}}{0.5 \rho_{0} U_{\infty}^{2}} \text { and } C_{p_{\text {wall }}^{R M S}}^{R M}=\frac{\sqrt{\overline{p_{\text {wall }}^{\prime 2}}}}{0.5 \rho_{0} U_{\infty}^{2}}
$$

where an overbar indicates the time average and a prime is related to the fluctuating pressure field. The pressure coefficient $C_{p_{\text {wall }}}$ increases as the flow velocity decreases (see Fig. 4). The maximum value is obtained at the corner of the step. This coefficient drastically decreases in the separation region located above the step. By comparing with previous $3 \mathrm{D}$ results $[16,20]$, a similar tendency is observed but the amplitude levels are quite different. This is directly related to the $2 \mathrm{D}$ character of present simulation. Moreover, the streamwise evolution of $C_{p_{\text {wall }}}^{R M S}$ coefficient follows a classical behavior $[16,20]$. The wall pressure fluctuation begins to increase from $x / h=-3$ and has its maximum value $(x / h \approx 1)$ just downstream the corner of the step and before the reattachment point. Then wall pressure fluctuations decrease due to the flow relaxation process following reattachment region. As it was previously observed [9], present results confirm that 

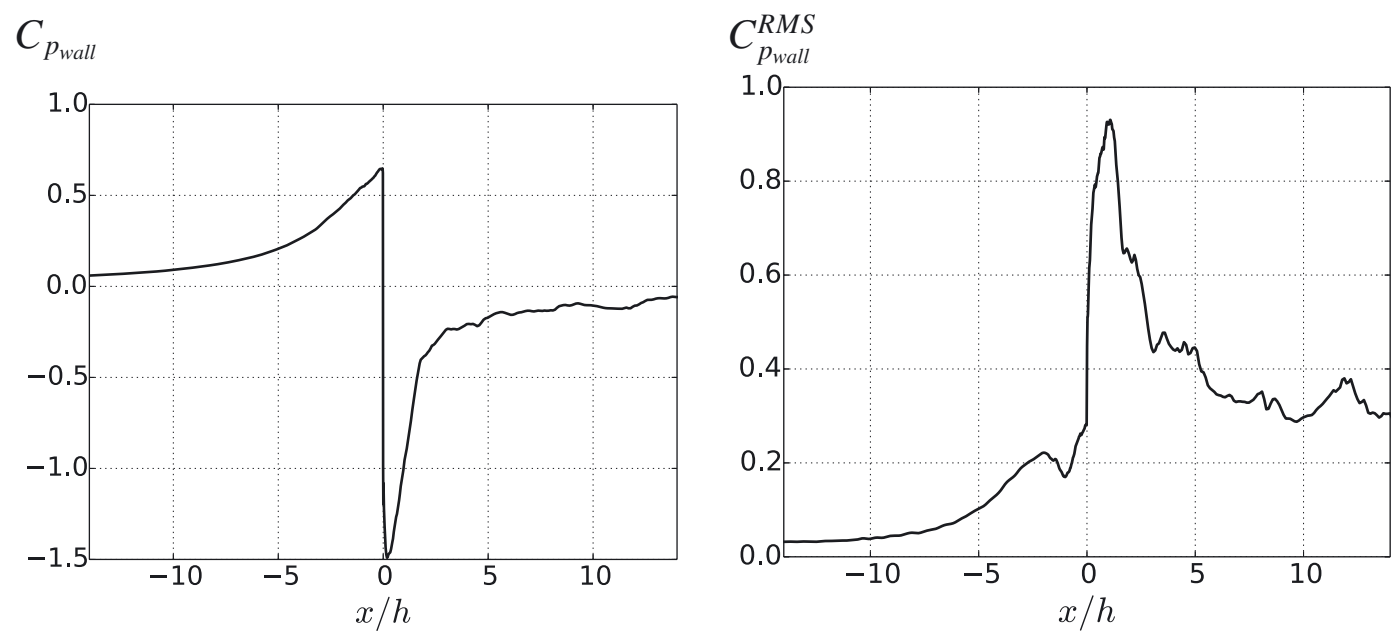

Fig. 4. Streamwise evolutions of the wall pressure coefficient $\left(C_{p_{\text {wall }}}\right.$, left hand side) and of the fluctuating wall pressure coefficient $\left(C_{p_{\text {wall }}}^{R M S}\right.$, right hand side).
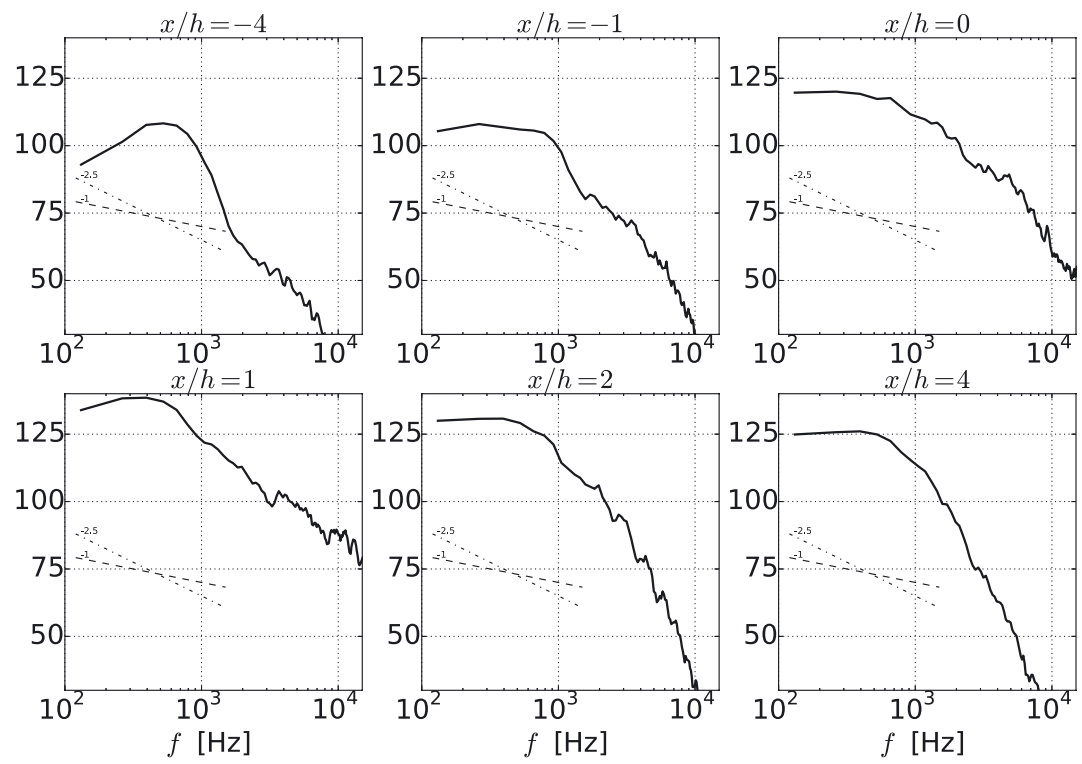

Fig. 5. Power Spectra Densities (dB scale, ref $4 \times 10^{-10} \mathrm{~Pa}^{2} \cdot \mathrm{Hz}^{-1}$ ) computed at selected $x$ locations.

the flow reattachment zone is of higher amplitude than the one of other zones. It may be a main source for the structure excitation.

A spectral analysis of the wall pressure is conducted to elucidate the spectral behavior of the wall pressure field. Figure 5 shows the power spectral density for six different pressure signals computed by the Welch periodogram method. The signals are extracted at selected $x$-locations related to different areas: (i) far upstream the step - TBL flow; (ii) near the corner of the step; (iii) in the shear layer flapping motion area; (iv) far downstream the recirculation region. First, it is shown that the spectrum magnitudes of the wall pressure in the recirculation area are higher than the other wall pressure spectrum ones. Second, each frequency spectrum exhibits a low frequency high magnitude region followed by two successive power law decay regions with similar slopes of -1 and
-2.5. This is in agreement with previous works dealing with similar flow configurations [16,20].

\subsection{Far acoustic pressure field analysis}

In the far-field acoustic $(y / h=20)$, the frequency spectra are broadband for frequencies lower than $f_{0}=$ $1000 \mathrm{~Hz}$. For higher frequencies $\left(f>f_{0}\right)$, a power law decay region is observed with a slope of -2.5 [33]. Such a frequency corresponds to a Strouhal number $S_{t}=f_{0} h / U_{\infty}$ of 0.5 . To determine the nature of the acoustic sources, the directivity patterns at $r=10 h$ (circle of radius $r$ centered at the corner of the step) of the Root Mean Square (RMS) of the acoustic pressure field are presented in Figure 6. This figure presents: (i) the directivity of the original acoustic pressure field, (ii) the directivity of the 


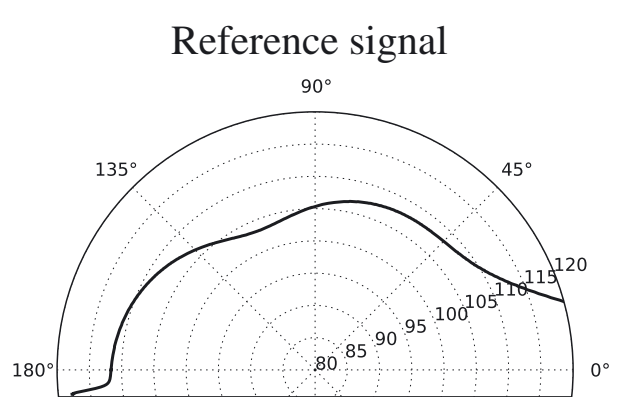

Filtered signal

$(f \in[0: 1000] \mathrm{Hz})$

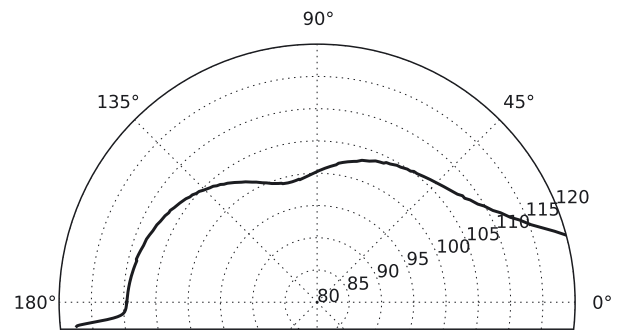

Filtered signal

$(f \in[1000: 5000] \mathrm{Hz})$

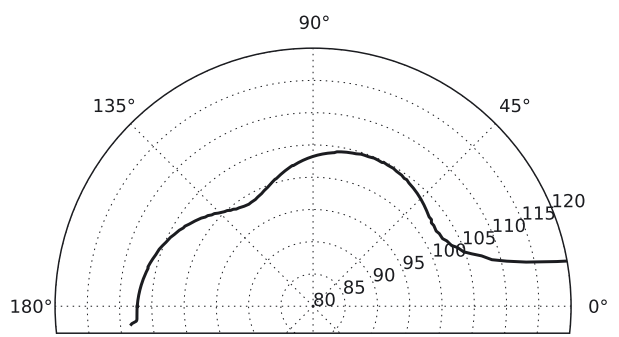

Fig. 6. Directivity patterns of the acoustic fluctuations at $r=10 h$ for selected filtered frequency bandwidth.

Table 1. Notations used in the following for the five test cases based on different inflow wall pressure fields. Each $p_{i}^{\text {method }}$ wall pressure field, with $i=1,2$ and method $=F T$ or $P O D$, will be defined in the following.

\begin{tabular}{cccccc}
\hline Test Case & 1 & 2 & 3 & 4 & 5 \\
\hline $\begin{array}{c}\text { Inflow wall pressure field } \\
\text { used for the next beam } \\
\text { and acoustic computations }\end{array}$ & Reference wall pressure & $p_{1}^{F T}$ & $p_{2}^{F T}$ & $p_{1}^{P O D}$ & $p_{2}^{P O D}$ \\
\hline
\end{tabular}

low-frequencies filtered pressure field $(f \in[0: 1000] \mathrm{Hz})$, (iii) the directivity of the middle-frequencies filtered pressure field $(f \in[1000: 5000] \mathrm{Hz})$. More than 3000 time steps are used to compute the RMS of acoustic pressure field. The directivity pattern of the RMS of the acoustic pressure indicates a pronounced effect for angle in $\left[140^{\circ}: 180^{\circ}\right]$, especially for the low pass filtered pressure field. For the middle-frequency filtered pressure field, two main pronounced effects are recovered for an angle around $80^{\circ}$ and for an angle in $\left[140^{\circ}: 180^{\circ}\right.$. This last result may be related to a dipolar nature of the source as previously noted in a similar 3D flow configuration [18]. Globally, even though some pronounced peaks may be observed in the directivity pattern which are quite similar to those previously obtained $[18,36]$ it is not possible to properly state about the nature of acoustic sources in such a flow configuration.

\subsection{Conclusive remark}

Even though the two-dimensional approach of the problem is a limited modeling, the main features of the physics of a FFS are recovered. This is then sufficient for the purpose of the present study that is to test the effectiveness of POD and Fourier techniques in such a fluid-structure-acoustic interaction problem.

\section{Flow decomposition analysis of the forward-facing step flow}

In this section, advanced post-processing mathematical tools are implemented to analyze and to decompose the wall pressure field, $p\left(x_{v i b}, t\right)$. The classical discrete fourier transform (referred to as FT in the following) and the proper orthogonal decomposition $(P O D)$ are successively considered for the investigation.

In the following, the reference wall pressure field extracted on the step will be linearly decomposed into two parts either by FT method or POD one. Consequently, $F T$ and $P O D$ applications lead to obtain 4 partial wall pressure fields: 2 related to a POD decomposition and 2 related to a Fourier decomposition of the reference wall pressure signal. These four pressure fields will be denoted: $p_{i}^{\text {method }}$ with $i=1,2$ and method $=F T$ or POD. Then, five test cases will be successively done to examine the fluid-structure-acoustic interaction problem as a function of the inlet wall pressure field. The notations given in Table 1 will be used in the following.

\subsection{Brief description of proper orthogonal decomposition (POD)}

$P O D$ is an efficient statistical technique providing an optimal decomposition in representing the flow variable 

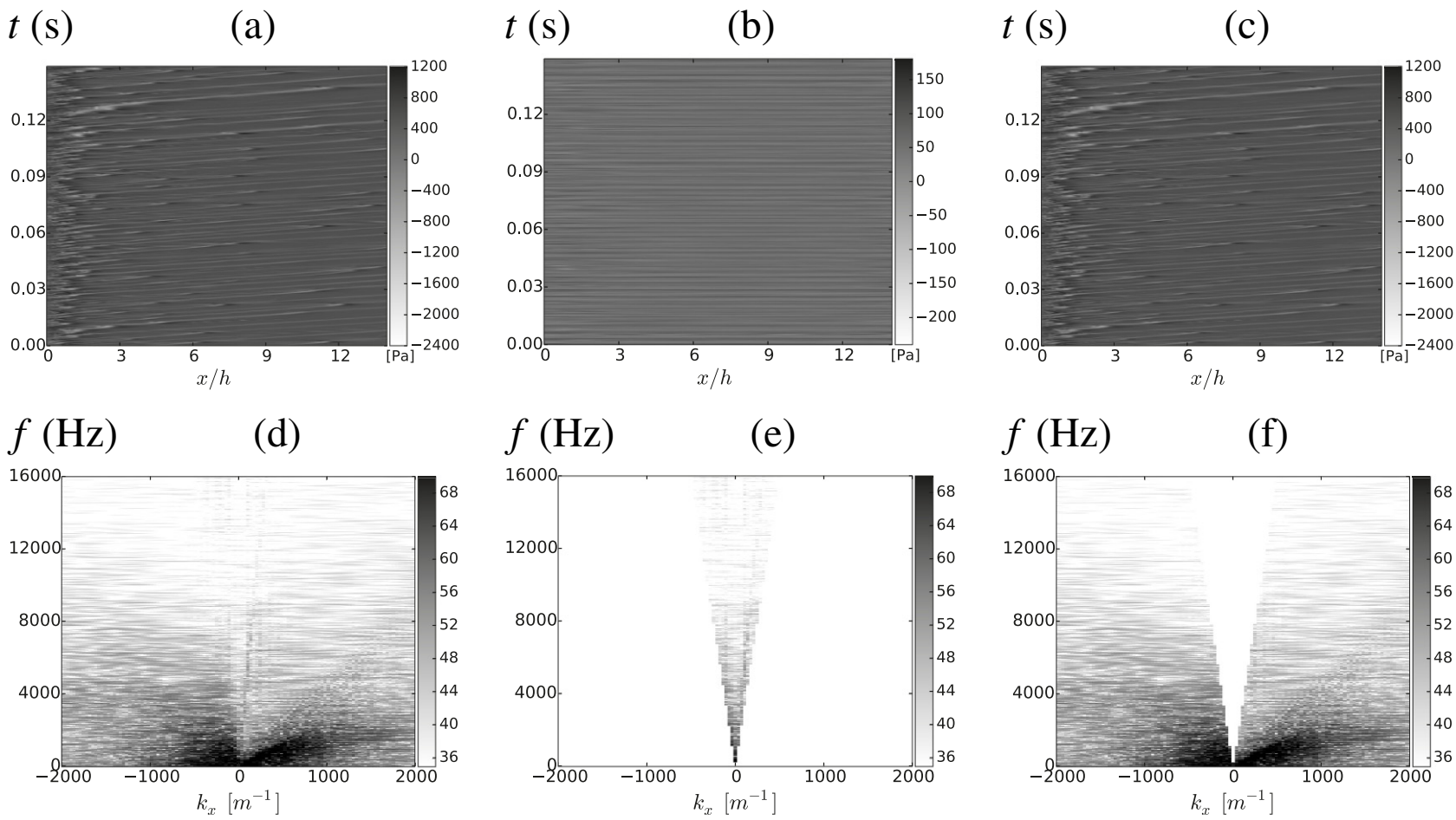

Fig. 7. Representation of the wall pressure signal: Top, in the physical space $(t, x / h)$ expressed in Pa. Bottom, in the wavenumber-frequency domain $\left(f, k_{x}\right)$, expressed in $\mathrm{dB}$ scale. Left: reference wall pressure field. Center: low wavenumber filtered field (acoustic component) and Right: high wavenumber filtered field (hydrodynamic component).

energy (here the energy is related to the RMS of the fluctuating pressure field). This maximization leads to a Fredholm integral eigenvalue problem [25] which consists in determining the spatial discrete orthogonal eigenfunctions denoted $\Phi^{(n)}$ of the two point spatial correlation tensor $R$ of the random pressure field:

$$
\int_{\mathcal{D}} R\left(\mathbf{X}, \mathbf{X}^{\prime}\right) \Phi^{(n)}\left(\mathbf{X}^{\prime}\right)=\lambda^{(n)} \Phi^{(n)}(\mathbf{X})
$$

$\lambda$ is the eigenvalue and $\mathcal{D}$ is the spatial domain under investigation. Using such $P O D$ basis, each instantaneous fluctuating pressure field can be expressed as a linear combination of $P O D$ eigenfunctions:

$$
p(\mathbf{X}, t)=\sum_{n=1}^{N_{\text {modes }}} a^{(n)}(t) \Phi^{(n)}(\mathbf{X}),
$$

where $a^{(n)}(t)$ are the random time coefficients of projection satisfying $a^{(n)}(t) a^{(m)}(t)=\delta_{n m}$ where $\delta$ is the Kronecker symbol. $N_{\text {modes }}$ is the total number of POD modes. The first $P O D$ modes capture most of the fluctuating pressure energy of the flow with a minimum of terms, compared to any flow decomposition. That explains the great development of $P O D$ application to turbulent flows due to its efficiency in extracting the large scale coherent structures present in turbulent flows $[37,38]$.

\subsection{Analysis of the wall pressure decomposition}

The wall pressure field stored data consists of 5000 instantaneous snapshots sampled at $f_{e}=33630 \mathrm{~Hz}$ with a sampling resolution of $\Delta f=6.7 \mathrm{~Hz}$. For post-processings, the wall pressure signal has to be available on a regular $x$-mesh grid. In this sense, to avoid any effect of the interpolation onto post-processing tools, a wall pressure signal discretized on the largest mesh step discretization is retained: $d x / h=1 / 10$. Then $N_{s}=140$ points are regularly extracted from the step wall.

Figure 7 (graphs (a), (b) and (c) displays the wall pressure field in the physical space $p\left(x_{v i b}, t\right)$ and in the spectral space $\hat{p}\left(k_{x}, f\right)$ where $k_{x}$ is the wavenumber along the streamwise direction. The physical representation is quite similar to the one given in reference [17]. For $x / h<1$, the great unsteady character of the flow is observed revealing the high pressure fluctuation footprints in the shear layer flapping motion area. When approaching the reattachment point $x / h \approx 2$, the highest pressure fluctuations are observed. Far upstream $(x / h>3)$, the space-time convection of the large scale flow structures are clearly indicated. The spectral representation exhibits the signature of these large scale flow structures that convect at $25 \mathrm{~m} . \mathrm{s}^{-1}$. This aerodynamic contribution is mainly observable at low frequencies $(f<1000 \mathrm{~Hz})$ and has a higher amplitude than the acoustic contribution related to low wavenumbers. The main characteristics of the present wall pressure field can then be regarded as similar to the ones of the wall pressure field impacting a side car window $[2,8]$. 
Mathematical tools are now implemented to decompose the wall pressure field as follows:

$$
p\left(x_{v i b}, t\right)=p_{1}^{\text {method }}\left(x_{v i b}, t\right)+p_{2}^{\text {method }}\left(x_{v i b}, t\right)
$$

where the method can be either FT or POD. According to mathematical tool properties, $p_{1}$ and $p_{2}$ pressure fields will differ in

- their wavenumber-frequency content application);

- their energy content (POD application).

\subsubsection{Fourier analysis of the wall pressure field}

The wall pressure field is of aeroacoustic nature. It contains an acoustic component and an aerodynamic one. The acoustic part propagates at the sound velocity while the aerodynamic component convects at the flow velocity, about $25 \mathrm{~m} \cdot \mathrm{s}^{-1}$. As phase velocity $c$ is linked to frequency $f$ and wavenumber $k$ by the relation $c=\frac{2 \pi f}{k}$, the acoustic contribution is associated, for a selected frequency, to the low wavenumbers. A spatiotemporal FT can then be used to distinguish both contributions of the wall pressure field as it was previously done [3]. Practically, one considers that the component convecting at a flow velocity superior to $200 \mathrm{~m} \cdot \mathrm{s}^{-1}$ cannot be associated with an aerodynamic component. It is then assumed to correspond to the denoted acoustic component. More precisely, the choice of the threshold convection velocity value is directly linked to the wavenumber and frequency resolutions. Here, the wavenumber resolution is $35.3 \mathrm{~m}^{-1}$ and the frequency resolution is $6.7 \mathrm{~Hz}$. Thus, for the first wavenumber $k=35.3 \mathrm{~m}^{-1}$, two flow events convected at $300 \mathrm{~m} \cdot \mathrm{s}^{-1}$ or $200 \mathrm{~m} \cdot \mathrm{s}^{-1}$ correspond to a frequency of $1686 \mathrm{~Hz}$ or $1124 \mathrm{~Hz}$ respectively. Present high wavenumber discretization induces then a condition on the determination convection velocity for the FFT filtering procedure. Indeed, to keep the low-wavenumber part of the acoustic field, one prefers retaining a convection velocity of $200 \mathrm{~m} \cdot \mathrm{s}^{-1}$ to discriminate both acoustic and aerodynamic contributions. In this sense, to extract this acoustic component, a triangle area is isolated in the Fourier space (see Fig. 7e). The three points delimiting the triangle area are $\left(k_{x}, f\right)=(0,0),\left(k_{x}, f\right)=(500,16000)$ and $\left(k_{x}, f\right)=(-500,16000)$. Then for the filtering of the acoustic component, one first extracts the spectral content only contained in this area, and then one performs an inverse FFT to retrieve the acoustic component of the signal in the physical domain. To access the aerodynamic part, the inverse $F T$ is computed over the remaining area. By applying such a filter, $\hat{p}\left(k_{x}, f\right)$ is then decomposed based on the summation of $\hat{p}_{1}^{F T}\left(k_{x}, f\right)$ and $\hat{p}_{2}^{F T}\left(k_{x}, f\right)$ pressure fields. In this case, pressure spectrum $\hat{p}_{1}^{F T}$ is mainly related to the acoustic component of $\hat{p}\left(k_{x}, f\right)$ while $\hat{p}_{2}^{F T}$ is mainly related to the aerodynamic one. An illustration of the filtering process is given in Figure 7. By realizing an inverse $F T$, the $\left(x_{v i b}, t\right)$ space representation of $p_{1}^{F T}\left(x_{v i b}, t\right)$ and of $p_{2}^{F T}\left(x_{v i b}, t\right)$ is obtained. These last two pressure fields are also displayed in Figure 7 (graphs

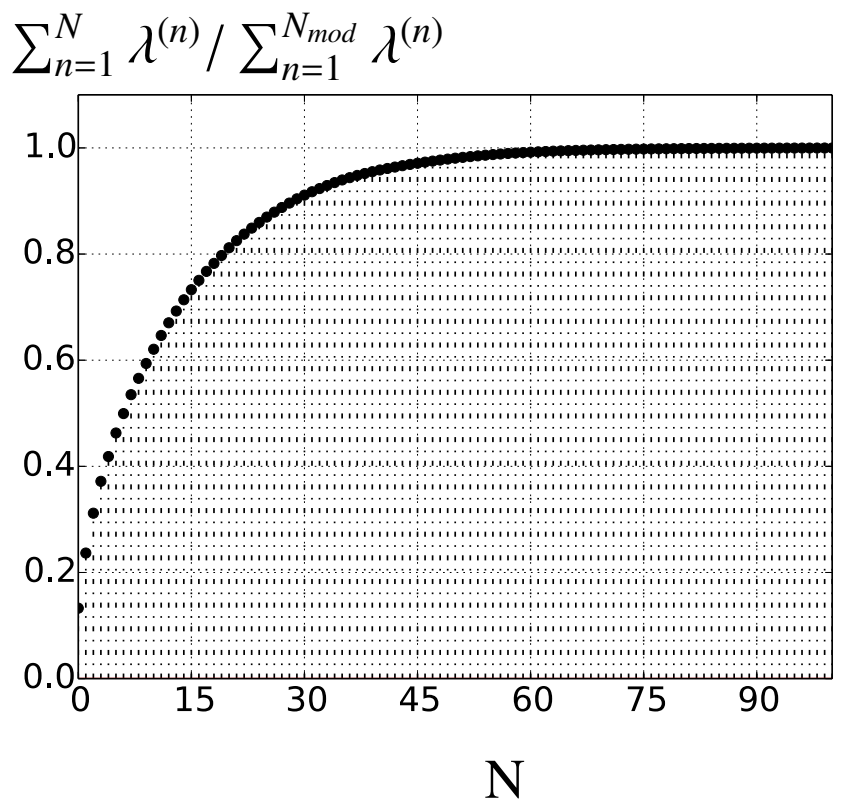

Fig. 8. $P O D$ mode energy convergence. $X$-axis is voluntarily limited to 100 .

(e) and (f)). The amplitude of $\hat{p}_{1}^{F T}\left(k_{x}, f\right)$ (acoustic component) is quite smaller than the one related to $p_{2}^{F T}$. The energy of the pressure signal is mainly contained in the energetic aerodynamic component of the reference signal. As observed in Figure 7 (graphs (b) and (c)) in the physical space, the amplitudes of $p_{1}^{F T}\left(x_{v i b}, t\right)$ and $p_{2}^{F T}\left(x_{v i b}, t\right)$ differ by a factor of 10 . These two filtered pressure fields will be separately used in Section 5 allowing the investigation of their associated radiated acoustic fields in the open cavity.

\subsection{2 $P O D$ analysis of the wall pressure field}

To perform a direct comparison with previous FFT analysis, the POD is implemented to the same wall pressure data. This simplified 1D stored data is also retained due to the fact that in the recirculation area, some most energetic flow events are observed compared to those observed downstream of this area. Based on such data, it seems easier to differentiate both flow events using the POD energetic flow partitioning. As the number of available grid points is small compared to the number of snapshots, the classic $P O D$ formulation introduced by Lumley [39] is used. That leads to obtain $N_{\text {modes }}=N_{s}$ (see Eq. (7)). An analysis of the POD mode convergence similar to the one detailed in reference [40] has been conducted [33]. This analysis shows that the first $10 P O D$ modes at least are statistically converged. Figure 8 presents the $P O D$ mode convergence. The first 4 cumulated $P O D$ modes contain $36 \%$ of the total fluctuating pressure energy respectively.

Figure 9 displays selected $P O D$ eigenfunctions $\Phi^{(n)}\left(x_{v i b}\right)$. Figure 10 represents the Fourier spectra of 

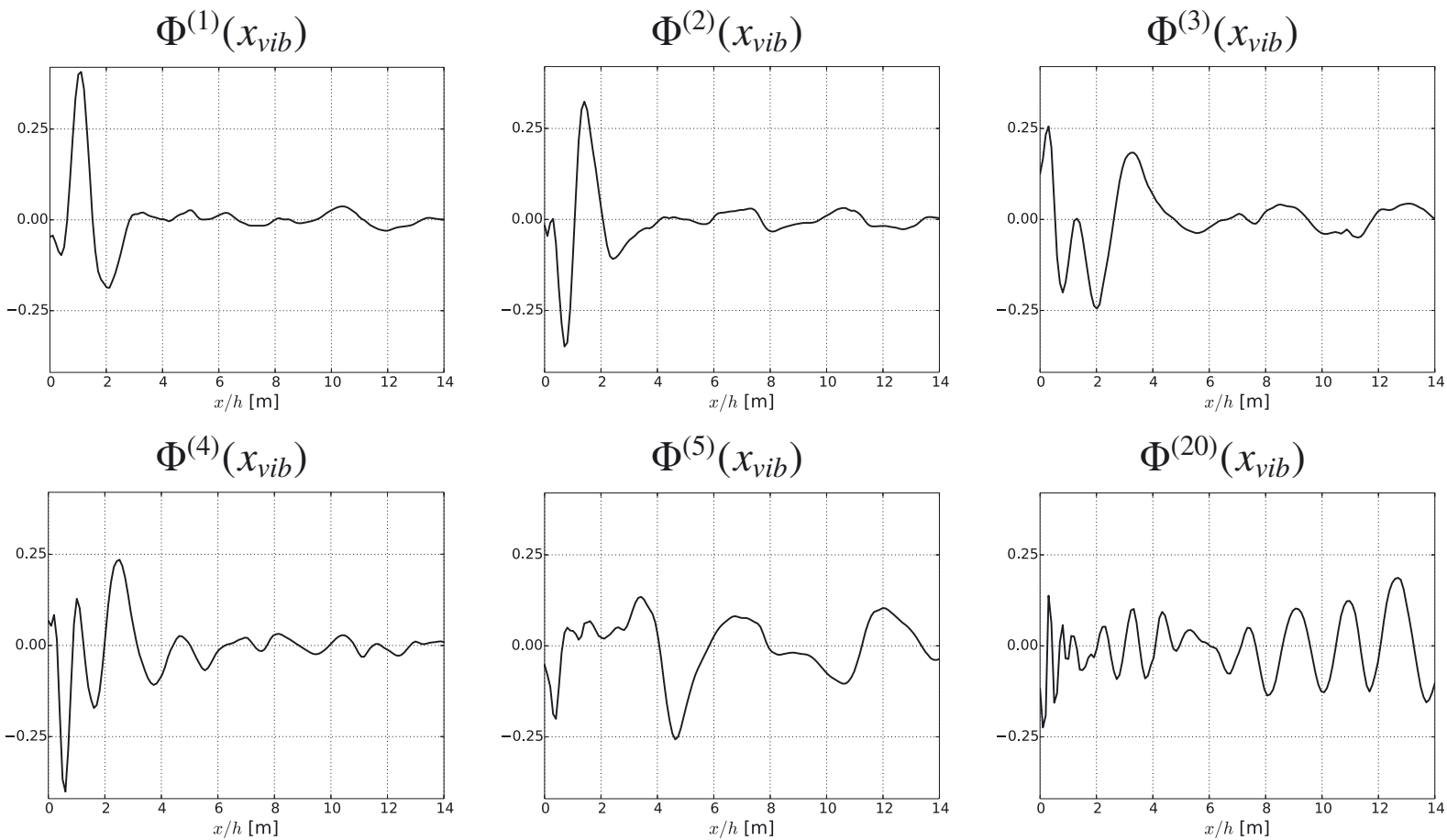

Fig. 9. Spatial evolution of the first 5 spatial POD eigenfunctions and also the 20 th one.

Mode 1

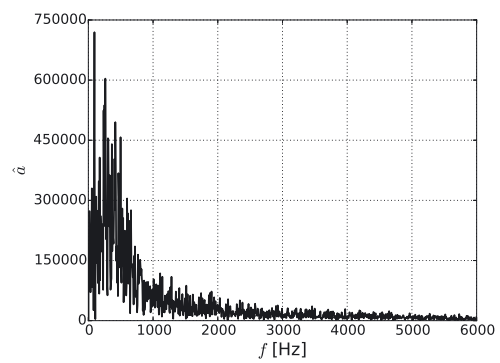

Mode 4

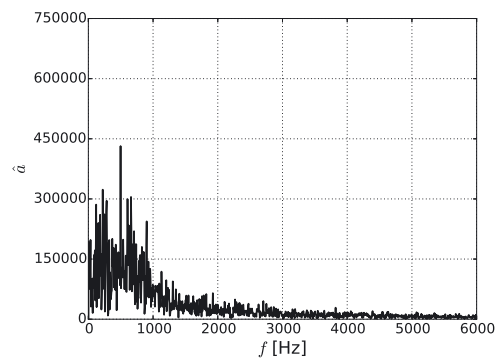

Mode 2

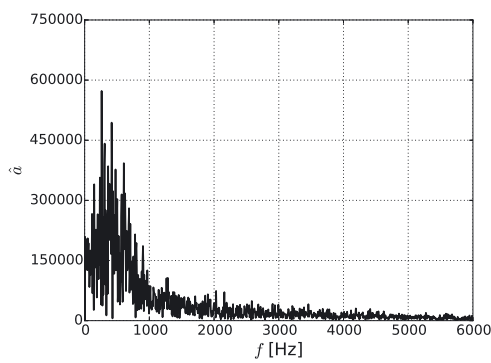

Mode 5

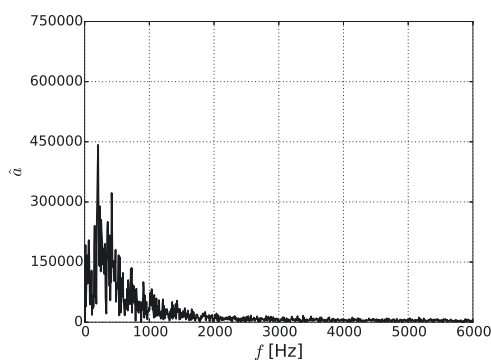

Mode 3

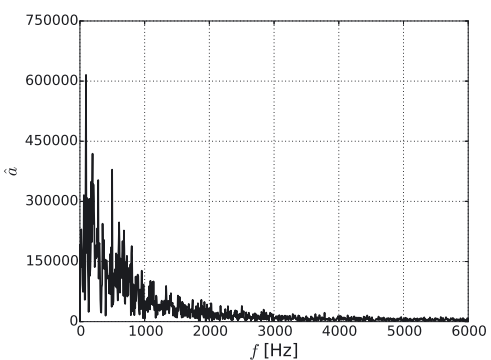

Mode 20

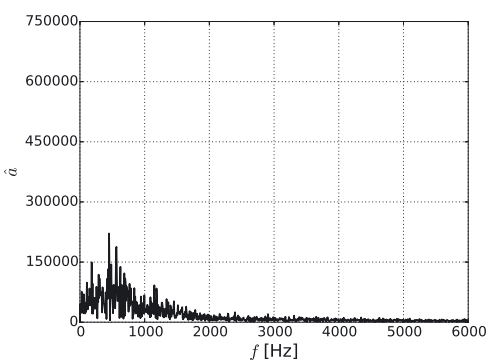

Fig. 10. Spectral representations of the first five temporal POD coefficients and also of the 20th one.

the first five temporal POD coefficients and also of the 20th one. Then, these first POD modes clearly extract the main energetic contribution of the flow, which is the one associated with the shear layer flapping instability $(x / h<2.5)$. Conversely when regarding high order POD modes, a high frequency component is extracted in the recirculation area and a lower frequency component is retrieved far away $(x / h>5)$ with a similar amplitude [33].
These high order POD modes are then mainly related to the eddy structure interacting with the wall far downstream the shear layer flapping motion. To select in an objective way, a POD cut-off mode number allowing the separation of organized motions and background turbulent part remains often questionable. It does not exist a universal criterion for such an investigation. However, it is generally expected that the first POD modes contain 

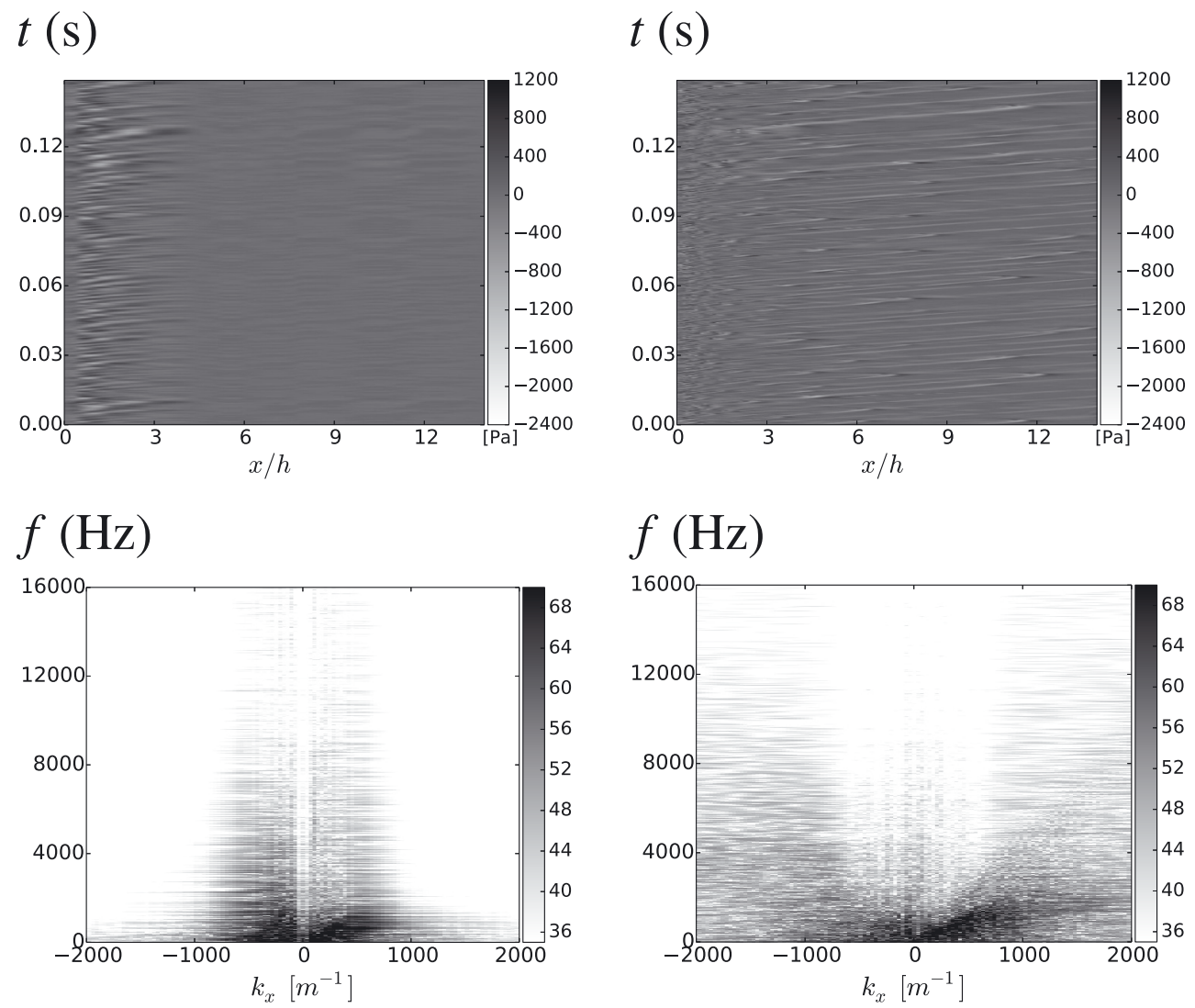

Fig. 11. Representation of the wall pressure signal: Top, in the physical space $(t, x / h)$ expressed in Pa. Bottom, in the wavenumber-frequency domain $\left(f, k_{x}\right)$, expressed in dB scale. Left: $p_{1}^{P O D}\left(x_{v i b}, t\right)$ and Right: $p_{2}^{P O D}\left(x_{v i b}, t\right)$.

the main part of the energetic organized flow pattern of the flow. In this work, the objective is to isolate the energetic vortex in the recirculation area and after several trials, it seems that the combination of the first four POD modes provides a nice extraction of these vortices. Using $P O D$ basis, the instantaneous wall pressure field is then decomposed following Equation (8):

$$
\begin{aligned}
p_{1}^{P O D}\left(x_{v i b}, t\right) & =\sum_{n=1}^{4} a^{(n)}(t) \Phi^{(n)}\left(x_{v i b}\right) \\
\text { and } & \\
p_{2}^{P O D}\left(x_{v i b}, t\right) & =\sum_{n=5}^{N_{\text {modes }}} a^{(n)}(t) \Phi^{(n)}\left(x_{v i b}\right)
\end{aligned}
$$

In this context, the $p_{1}^{P O D}$ pressure field corresponds to the wall pressure field projected onto the first four POD modes and it is associated with the main energetic flow pattern of the wall pressure field. The $p_{2}^{P O D}$ field (called the residual part of the wall pressure field) corresponds to the wall pressure field projected onto the POD mode remainder and it is associated with the low energetic flow pattern of the wall pressure field. Note that the purpose of the current $P O D$ application is not to discriminate acoustic and aerodynamic parts of the pressure field as previously tested $[8,26]$. Indeed, POD application is optimal in an energetic context and then a specific POD mode may also contain information about several distinct flow events (energetic or not). In this context, POD cannot be viewed in the present study as a method to interpret the acoustic source terms. In this study, contrary to FT filtering decomposition, this method offers a new way for decomposing the wall pressure field based on an energetic criterion. Figure 11 illustrates the resulting $P O D$ flow decomposition by plotting $p_{1}^{P O D}\left(x_{v i b}, t\right)$ and $p_{2}^{P O D}\left(x_{v i b}, t\right)$ pressure fields. The first $P O D$ modes clearly extract favorably the energetic wall pressure field in the recirculation area. The residual part (pressure field $p_{2}^{P O D}$ ) exhibits a low energetic vortex signature in the downstream area $(x / h<3)$. This last contribution is then mainly related to the convected flow structures interacting with the wall after the recirculation area. Spectral representations show that the acoustic component is shared by $p_{1}^{P O D}$ and $p_{2}^{P O D}$.

\section{Analysis of the structure vibro-acoustic response and of the acoustic radiation in the cavity}

The vibroacoustic response of the wall pressure loading is now investigated. The properties of the vibrating structure have already been given (see Sect. 2.2). As a preliminary result, the first radiated modes of the vibrating structure are computed theoretically. They are given 
Table 2. Theoretical structural modes of the vibrating glass structure.

\begin{tabular}{cccccccc}
\hline Mode number & 1 & 2 & 3 & 4 & 5 & 6 & 7 \\
\hline Frequency [Hz] & 164 & 471 & 905 & 1517 & 2287 & 3160 & 4236 \\
Wavenumber $k_{s}$ & 26 & 44 & 61 & 79 & 97 & 114 & 132 \\
Wavenumber $k_{c}$ & 41 & 118 & 227 & 381 & 575 & 794 & 1065 \\
Wavenumber $k_{0}$ & 3 & 9 & 17 & 29 & 43 & 60 & 80 \\
\hline
\end{tabular}

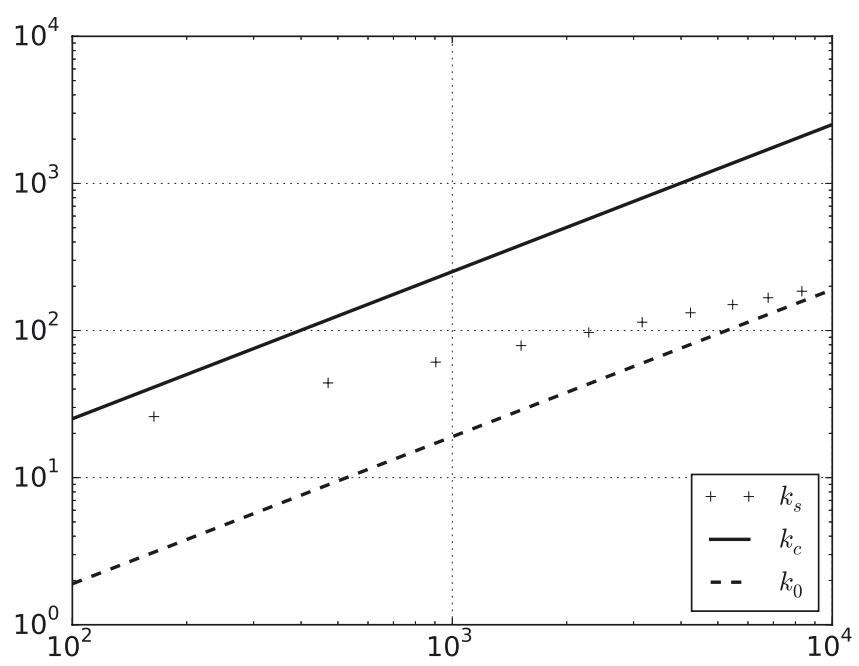

Fig. 12. Theoretical structural modes of the vibrating glass structure.

in Table 2 and presented in a graph in Figure 12. Turbulence convection wavenumber $k_{c}$ and acoustic wavenumber $k_{0}$ at the structural resonance frequencies are also shown. The hydrodynamic coincidence condition is below the first beam resonance frequency and the acoustic coincidence condition is above the seventh beam resonance frequency. This knowledge of the modal frequencies is of interest in the following interpretation of the vibroacoustic response.

\subsection{Beam solver response}

The reference wall pressure field $p\left(x_{v i b}, t\right)$ is used as an inflow condition to compute the reference velocity response. Recall that the present numerical simulation is based on a simplified coupling method between the pressure distribution and the flow field and the vibrating structure. It is not based on an interacting method but based on a weak coupling. However, note that previous tests [33] have been performed based only on one computation taking into account the vibro-acoustic coupling. Deduced results have demonstrated that the sound due to the vibrating structure itself has no effect on the FFS flow or on the radiated acoustic field in the cavity. An illustration of the beam solver computation is presented in Figure 13 (top) displaying the vibration velocity response. Each wall pressure field $p_{i}^{\text {method }}\left(x_{v i b}, t\right)$ (with $i=1,2$ and method $=F T, P O D)$ is also successively used as inflow conditions (see Tab. 1). The resulting vibration transverse velocities are represented in Figure 13. As a first observa-
Table 3. Global radiated energy (expressed in $\mathrm{dB}$ ) in the open cavity for each test case.

\begin{tabular}{cccccc}
\hline Test cases & 1 (Reference) & 2 & 3 & 4 & 5 \\
\hline Acoustic energy & 106.47 & 106.65 & 92.40 & 104.71 & 102.03 \\
\hline
\end{tabular}

tion, the amplitude of transverse velocity computed from the test case 2 is much smaller than the one computed from other test cases. This is directly related to previous comments (see Sect. 4.2.1) according to the content of $p_{1}^{F T}$ wall pressure field. Moreover, the results of the test case 3 approach the ones of the reference test case 1. That seems to argue that the aerodynamic component of the wall pressure field is mainly responsible for the structure vibration. On the other hand, even if the wall pressure $p_{1}^{P O D}$ field is less energetic than the $p_{2}^{P O D}$ one, the amplitude of resulting transverse velocity is quite similar in both cases. Nevertheless, the frequency contents of both beam solver responses differ. For the test case 4 , based on $p_{1}^{P O D}$ pressure field, a low frequency component seems to dominate in the vibration transverse velocity response. This behavior could be directly due to the low frequency component of the energetic flow structures associated with the shear layer flapping motion which is well extracted based on the first POD modes (see the spectral representations of the first POD temporal coefficients in Fig. 10). These large scale flow structure instabilities impact the wall regularly and lead to force the low frequency excitation of the vibrating structure.

\subsection{Acoustic radiation in the cavity}

The transverse velocity computed with the beam solver is now used as an inflow condition for the numerical simulation of the acoustic propagation in the cavity. As previously, five test cases are performed (see Tab. 1). As a first investigation, Table 3 gives the global radiated energy (expressed in $\mathrm{dB}$ ) in the open cavity. The acoustic energy values computed from the test case 2 differs from more than $20 \mathrm{~dB}$, to the one related to the test case 3 , demonstrating that the main acoustic energy in the cavity is related to the aerodynamic part of the wall pressure field. Indeed, this part allows the recovering of the whole acoustic energy. As for POD test cases 4 and 5, a quasi similar energy level is retrieved slightly inferior to the one of the reference test case 1 .

During each simulation when the flow is established, instantaneous acoustic pressure field is stored at the fixed point located exactly at the center of the cavity. Figure 14 displays the time evolution of the acoustic pressure signal and its associated spectral representation. To enhance the comparative analysis, the acoustic spectrum deduced from the reference test case is always superimposed onto the acoustic spectra deduced from other test cases.

Moreover, Figure 16 represents similar representations but for the acoustic signals stored at a point close to the middle of the beam. These two figures show quasi similar results detailed below. 
E. Gaudard et al.: Mechanics \& Industry 18, 201 (2017)

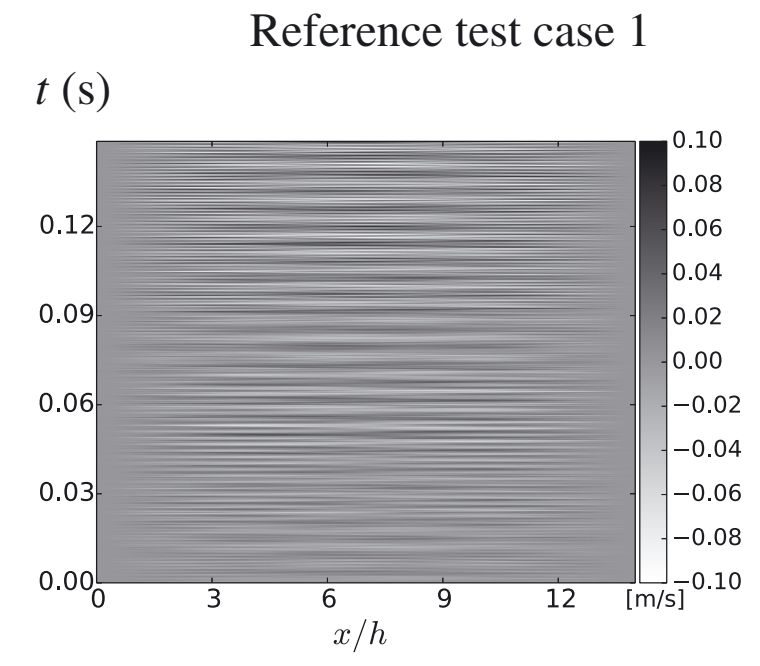

Test case 2

$t$ (s)

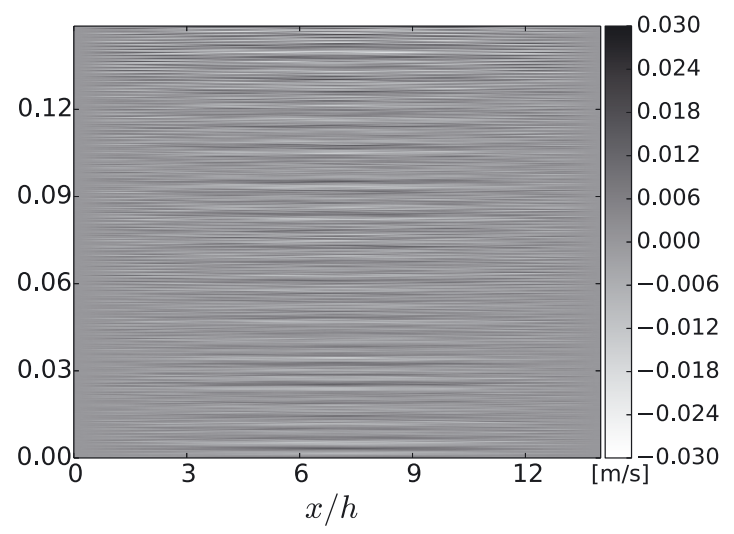

Test case 4

$t$ (s)

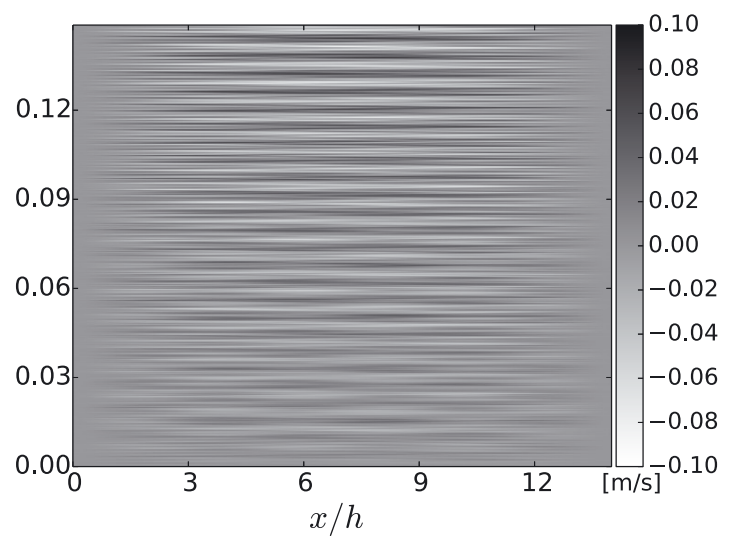

Test case 3

\section{$t$ (s)}

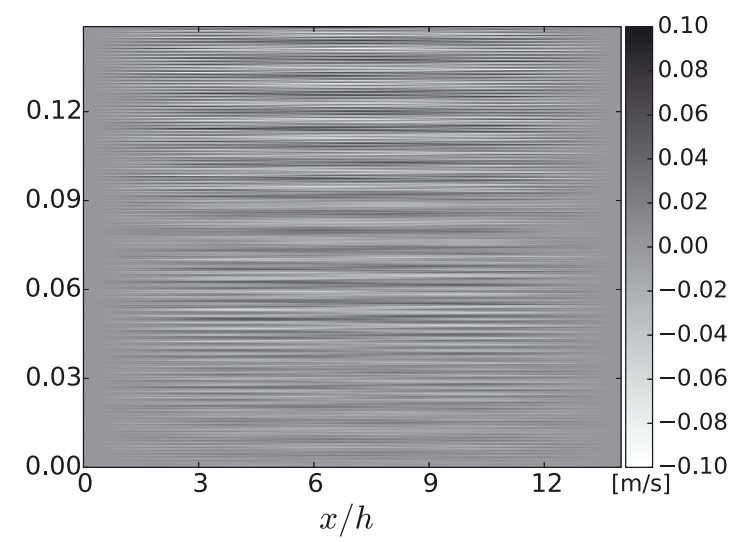

Test case 5

$t$ (s)

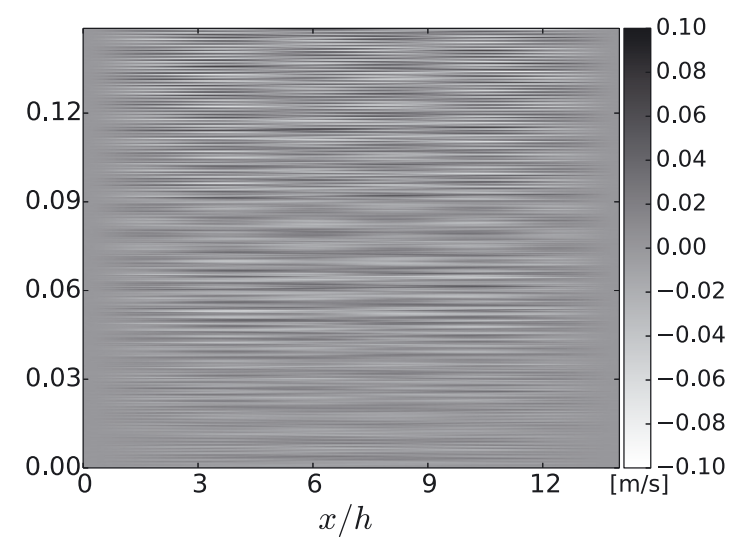

Fig. 13. Beam solver computation. Vibration transverse velocity response of the structure for different test cases (see Tab. 1). 
E. Gaudard et al.: Mechanics \& Industry 18, 201 (2017)

Test Case

\section{1: Reference}

2: $p_{1}^{F T}$

3: $p_{2}^{F T}$

4: $p_{1}^{P O D}$

5: $p_{2}^{P O D}$ time evolution
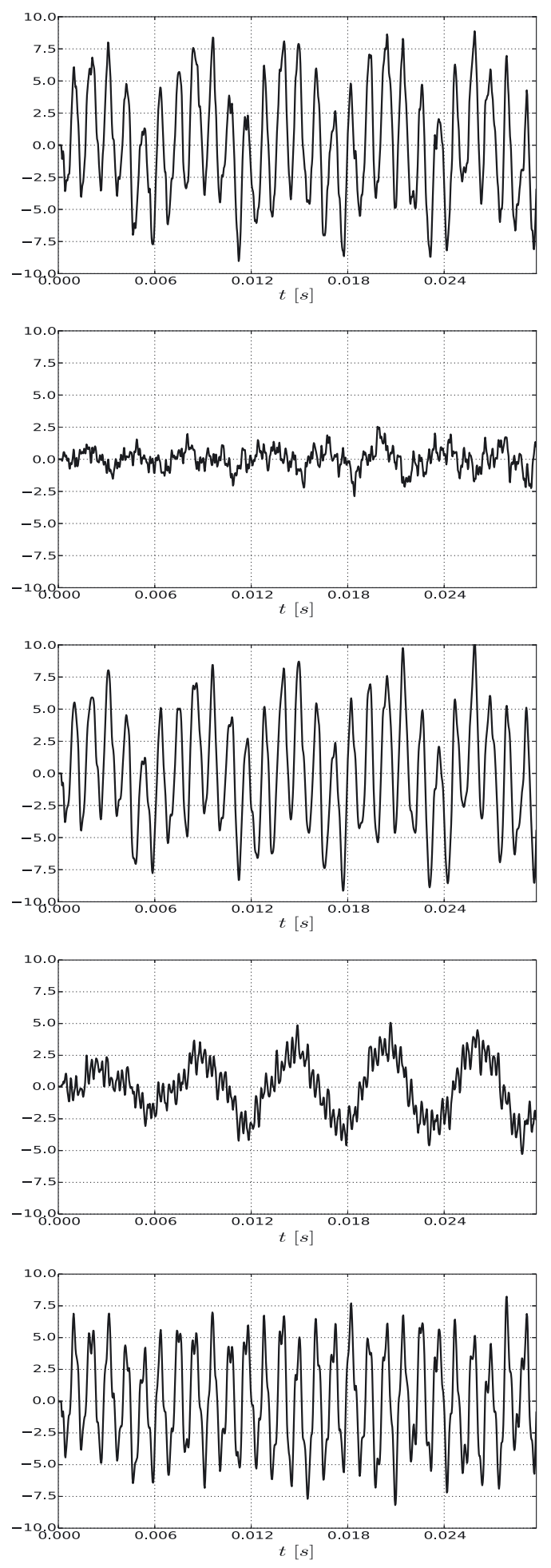

Spectral representation
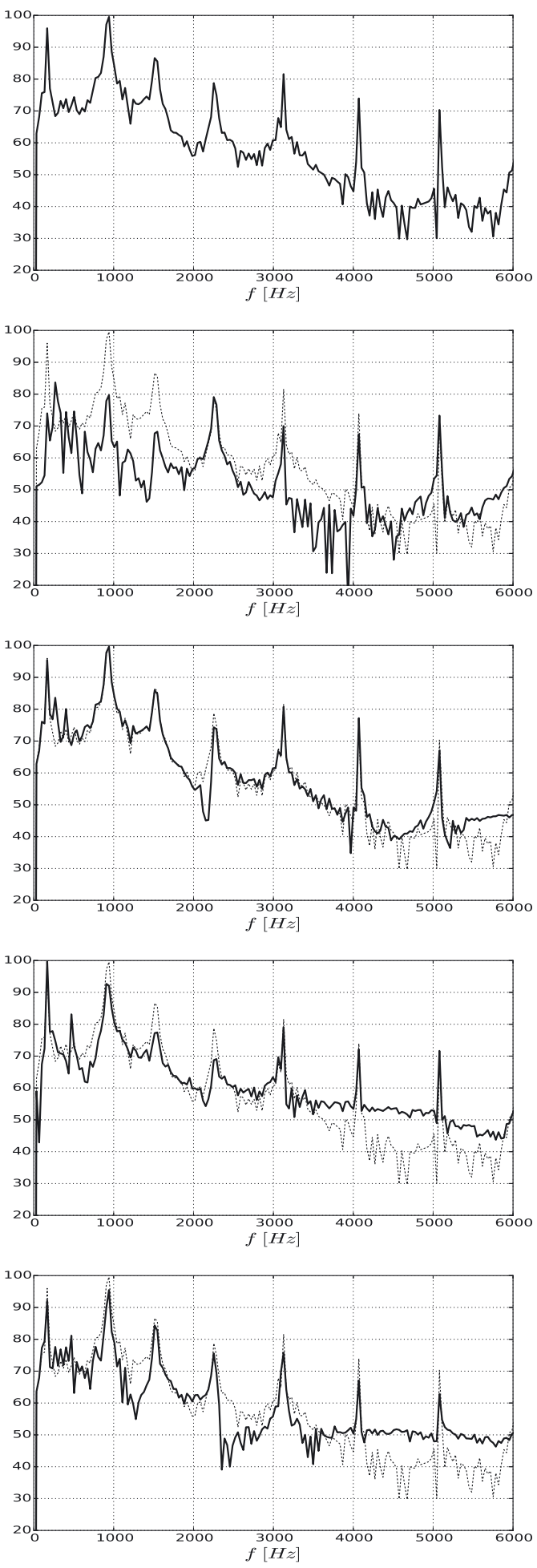

Fig. 14. Left: time evolution of the radiated acoustic pressure stored at the center of the cavity. Right: Corresponding Power Spectra Densities ( $\mathrm{dB}$ scale, ref $4 \times 10^{-10} \mathrm{~Pa}^{2} \cdot \mathrm{Hz}^{-1}$ ). In each spectral representation, the acoustic spectrum computed from the reference test case is also plotted (with dotted-line) for comparison. 
Several peaks appear in the acoustic spectrum computed from the reference test case. These peaks are well separated and they correspond to the structural modes of the vibrating wall (see Tab. 2). Note that to avoid any confusion about the dependence of the time duration for the post-processing, we performed similar post-processing analysis based on twice the current simulation time duration. Deduced results show that no real difference is noticeable compared to the present ones. Apart from structural mode number 2 , all the structural modes contribute to the acoustic response in the cavity. Structural modes 1 and 3 (that correspond to frequencies: $164 \mathrm{~Hz}$ and $904 \mathrm{~Hz}$ respectively) present a large magnitude and dominate the others. Note that it may not be possible to retrieve exactly the frequencies of the structural mode due to the dependence of frequency resolution imposed by the time duration of the simulation.

In each test case, the acoustic spectrum also shows peaks around the structural mode of the wall. However, in each case the amplitude of the frequency peak differs. According to results of test cases 2 and 3, the main noise contribution radiated in the cavity is due to the filtered aerodynamic pressure field, $p_{2}^{F T}$. Indeed, test case 3 exhibits a similar time evolution and spectral representation to the ones obtained with the reference test case. Even if the acoustic signal computed from test case 2 is of very low amplitude, the associated acoustic radiation in the cavity is non-negligible, especially for high frequencies (see Fig. 15), confirming previous statements [3]. It is noticeable to observe that the test case 2 shows that the beam mode of $2287 \mathrm{~Hz}$ is entirely recovered. It also contains each frequency observed in the reference test case. Moreover, as the FFT filtering procedure is quite dependent on the wavenumber-frequency resolution [26], it may be possible that the acoustic contribution is not entirely extracted based on the present filtering procedure especially at very low frequencies. Then such an acoustic wall pressure field could also have a higher contribution in the radiated acoustic pressure field in the cavity. Concerning the test cases 4 and 5, the acoustic pressure signal computed from the most energetic $p_{1}^{P O D}$ pressure field is of slightly higher amplitude than the one deduced from the test case $5\left(p_{2}^{P O D}\right.$ pressure field). The acoustic pressure fields from test cases 4 and 5 contain the same frequency peaks as the reference test case. The beam modes 4 and $5(1517 \mathrm{~Hz}$ and $2287 \mathrm{~Hz})$ are better restituted thanks to the test case $5\left(p_{2}^{P O D}\right.$ wall pressure field). Conversely, test case 4 exhibits a first frequency peak $(f \approx 164 \mathrm{~Hz}$ corresponding to the first beam mode) of several orders superior to the one related to the test cases 1 and 5 . It seems then that the structural vibration response leading to contribute to the first structural acoustic mode is quite related to the shear layer flapping motion that corresponds to the most energetic large scale flow structure. This result emphasizes the fact that the low frequency acoustic component obtained in the cavity is mainly associated with the recirculation area. Then, to reduce the low frequency acoustic noise in a similar flow configuration, one has to implement future control strategies

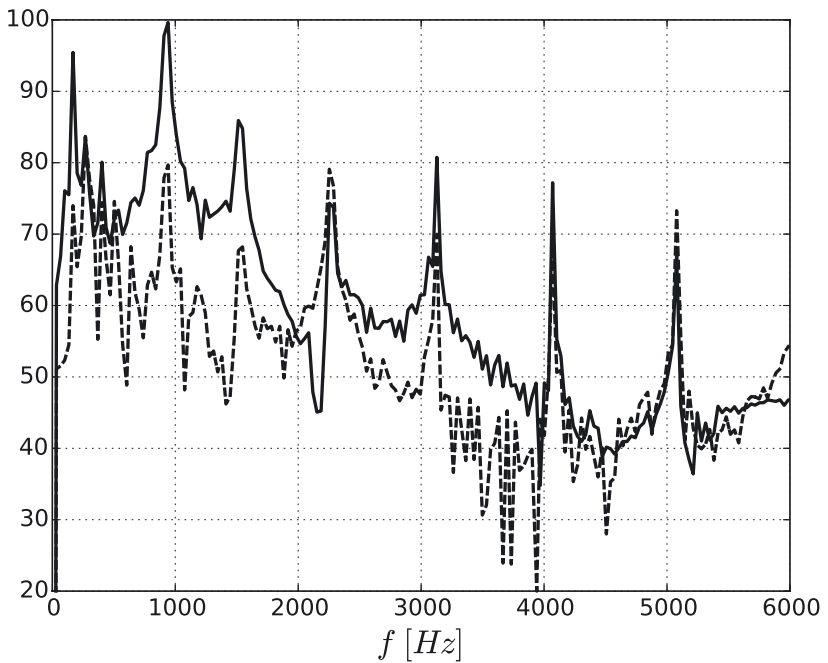

Fig. 15. Power Spectra Densities (dB scale, ref $4 \times$ $10^{-10} \mathrm{~Pa}^{2} \cdot \mathrm{Hz}^{-1}$ ) of the radiated acoustic due to the aerodynamic component (line) and due to the aeroacoustic component (dotted line).

allowing to reduce and/or limit the separated flow. For the investigation, POD could be used to control the flow separation [41].

\section{Conclusion}

The noise radiated by a vibrating structure into a cavity is of particular interest in modern automobile and aerospace industry. The numerical investigation of realistic flow configuration related to plane or vehicle applications remains quite complex today. In this sense, we propose in this work to consider a simplified flow configuration allowing the generation of similar fluid-structureacoustic interactions to those observed in realistic industrial application. A FFS flow configuration is retained and the upper part of the step is assumed to be a vibrating plate permitting the examination of radiated noise in an open cavity located under the plate. Three successive computations are performed. First, the numerical FFS flow characteristics are observed to be similar to the ones previously given in the literature even if a $2 \mathrm{D}$ flow configuration is considered. Second, based on each wall pressure field defined from FT or POD applications, the radiated acoustic pressure emitted in the cavity is determined. It is then observed that the main contribution of the interior noise is due to aerodynamic component extracted based on a frequency-wavenumber filtering procedure using FT. However, it is also confirmed that the acoustic pressure structural loading may have a non-negligible contribution in the radiated acoustic pressure. $P O D$ applications lead to the following results: (i) the most energetic wall pressure field corresponds to the shear layer flapping motion; (ii) this energetic flow field mainly contributes to the low frequency noise in the cavity; (iii) the $P O D$ flow partitioning allows the discrimination of two components 
Test Case

\section{1: Reference}

2: $p_{1}^{F T}$

3: $p_{2}^{F T}$

4: $p_{1}^{P O D}$

5: $p_{2}^{P O D}$ time evolution
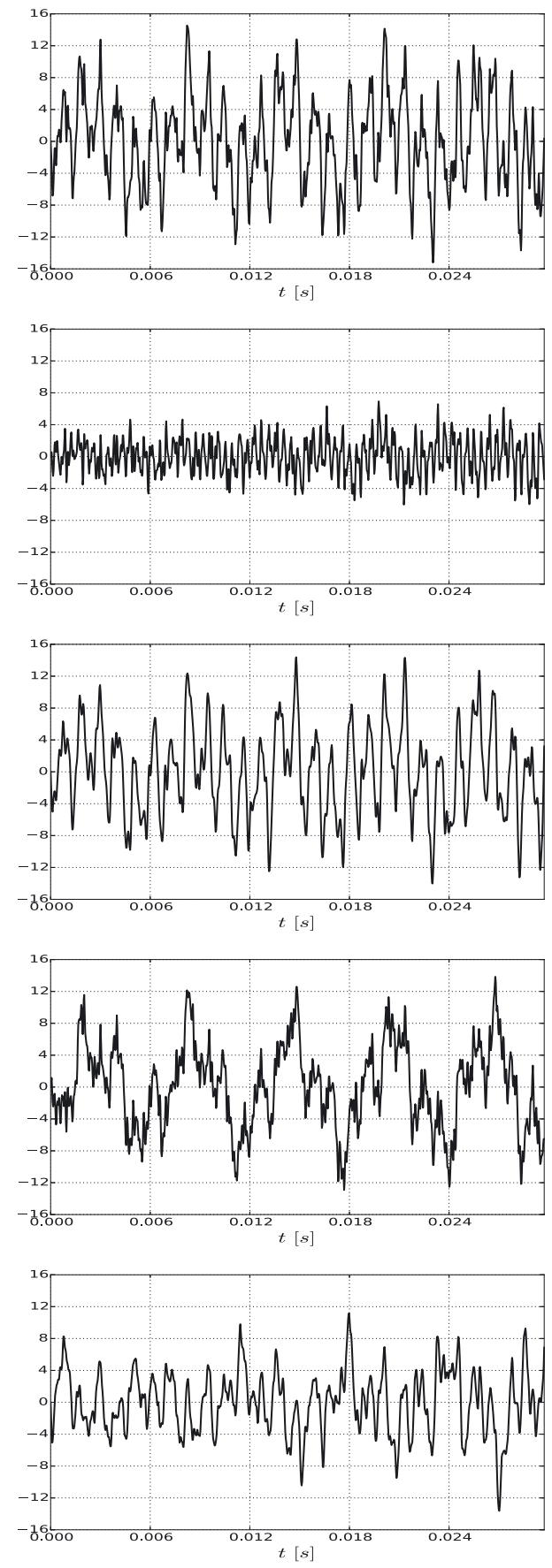

Spectral representation
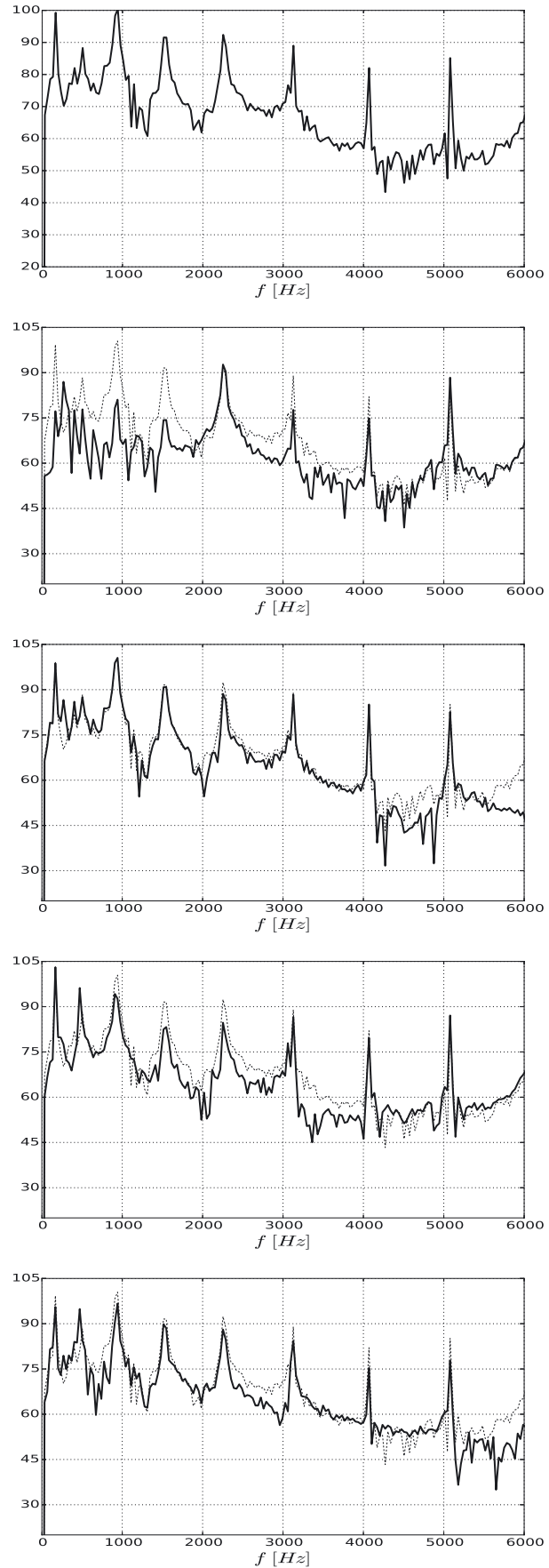

Fig. 16. Left: time evolution of the radiated acoustic pressure stored at the point close to the middle of the beam. Right: corresponding Power Spectra Densities (dB scale, ref $4 \times 10^{-10} \mathrm{~Pa}^{2} \cdot \mathrm{Hz}^{-1}$ ). In each spectral representation, the acoustic spectrum computed from the reference test case is also plotted (with dotted-line) for comparison.

which contribute in a similar manner to the noise radiated in the cavity.

This study permits to test the ability of two postprocessing mathematical tools to elucidate the aeroacoustic contribution of an unsteady flow which impacts a vibrating structure and associated with the main noise radiated in a cavity located under the structure. These post- processing tools offer then some great prospects for the future analysis of a more realistic fluid-structure-acoustic interaction problem occurring in automobile industry.

Based on these preliminary results obtained in a simplified 2D flow configuration, the potential of both mathematical tools has been emphasized. Thus, a prospect of this study is to perform a more realistic 
3D forward step flow configuration allowing to confirm these preliminary results. In this case, both FFT and POD methods will be implemented to decompose the $2 \mathrm{D}$ wall pressure field. The FFT and POD algorithms remain the same and have already been applied to similar $2 \mathrm{D}$ wall pressure field but without the same aim $[5,26]$. However, 3D applications need some great additional computational costs.

\section{References}

[1] F. Schafer, S. Muller, T. Uffinger, S. Becker, J. Grabinger, M. Kaltenbacher, Fluid-structure-acoustics interaction of the flow past a thin flexible structure, AIAA J. 48 (2010) $738-748$

[2] M. Hartmann, J. Ocker, T. Lemke, A. Mutzke, V. Schwarz, H. Tokuno, R. Toppinga, P. Unterlechner, G. Wickern, Wind noise caused by the a-pillar and the side mirror flow of a generic vehicle model, in: 18th AIAA/CEAS Aeroacoustic Conference, AIAA paper 2012-2205, 2012

[3] B. Arguillat, D. Ricot, C. Bailly, G. Robert, Measured wavenumber-frequency spectrum associated with acoustic and aerodynamic wall pressure fluctuations, J. Acoust. Soc. Am. 128 (2010) 1647-1655

[4] T. Bravo, C. Maury, A synthesis approach for reproducing the response of aircraft panels to a turbulent boundary layer excitation, J. Acoust. Soc. Am 129 (2011) 143153

[5] A. Hekmati, D. Ricot, Ph. Druault, Numerical synthesis of aeroacoustic wall pressure fields over a flat plate: Generation, transmission and radiation analyses, J. Sound Vib. 332 (2013) 3163-3176

[6] O. Robin, A. Berry, S. Moreau, S. Campeau, Experimental reproduction of random pressure fields for vibroacoustic testing of plane panels experimental reproduction of random pressure fields for vibroacoustic testing of plane panels, in: 19th AIAA/CEAS Aeroacoustic Conference, AIAA paper 2013-2027, 2013.

[7] W. Blake, Mechanics of flow-induced sound and vibration, Academic Press, London, 1986, Vols. I and II

[8] Ph. Druault, A. Hekmati, D. Ricot, Discrimination of acoustic and turbulent components from aeroacoustic wall pressure field, J. Sound Vib. 332 (2013) 7257-7278

[9] T.M. Farabee, M. Cassarella, Measurements of fluctuating wall pressure for separated/reattached boundary layer flows, J. Vib. Acoust. Stress Reliab. Design 108 (1986) 301-307

[10] J.F. Largeau, V. Moriniere, Wall pressure fluctuations and topology in separated flows over a forward-facing step, Exp. Fluids 42 (2007) 21-40

[11] M. Sherry, D. Lo Jacono, J. Sheridan, An experimental investigation of the recirculation zone formed downstream of a forward facing step, J. Wind Eng. Ind. Aerodynamics 98 (2010) 888-894

[12] T.M. Farabee, P.J. Zoccola, Experimental evaluation of noise due to flow over surface steps, in: ASME International Mechanical Engineering Congress and Exposition, 1998

[13] M. Jacob, A. Louisot, D. Juvé, S. Guerrand, Experimental study of sound generated by backwardfacing steps under wall jet, AIAA J. 39 (2001) 1254-1260
[14] S. Becker, M. Escobar, C. Hahn, I. Ali, M. Kaltenbacher, B. Basel, M. Grunewald, Experimental and numerical investigation of the flow induced noise from a forward facing step, in: 11th AIAA/CEAS Aeroacoustics Conference, AIAA paper 2005-3006 2005

[15] M.R. Catlett, Flow induced noise from turbulent flow over steps and gaps, Master's thesis, Virginia Polytechnic Institute and State University, 2010

[16] M. Ji, M. Wang, Sound generation by turbulent boundary-layer flow over small steps, J. Fluid Mech. 654 (2010) 161-193

[17] Y. Moon, J. Seo, Y. Bae, M. Roger, S. Becker, A hybrid prediction method for low-subsonic turbulent flow noise, Comput. Fluids 39 (2010) 1125-1135

[18] I. Ali, S. Becker, J. Utzmann, C. Munz, Aeroacoustic study of a forward facing step using linearized Euler equations, Physica D 237 (2008) 2184-2189

[19] C. Scheit, A. Esmaeili, S. Becker, Direct Numerical Simulation of flow over a forward-facing step - flow structure and aeroacoustic source regions, Int. J. Heat Fluid Flow 43 (2013) 184-193

[20] M. Awasthi, High Reynolds number turbulent boundary layer flow over small forward facing steps, Master's thesis, Virginia Polytechnic Institute and State University, 2012

[21] S. Glegg, B. Bryan, W. Devenport, M. Awasthi, Sound radiation from forward facing steps, in: 18th AIAA/CEAS Aeroacoustics Conference, AIAA paper 2012-2050, 2012

[22] M. Awasthi, J. Forest, M. Morton, W. Devenport, S. Glegg, The disturbance of a high Reynolds number turbulent boundary layer by small forward steps, in: 17th AIAA/CEAS Aeroacoustics Conference, AIAA paper 2011-2777, 2011

[23] D. Wilhelm, C. Hartel, L. Kleiser, Computational analysis of the two-dimensional-three-dimensional transition in forward-facing step flow, J. Fluid Mech. 489 (2003) 1-27

[24] H. Stüer, Investigation of separation on a forward facing step, Ph.D. thesis, Swiss Federal Institute of Technology Zürich, 1999

[25] P. Holmes, J. Lumley, G. Berkooz, Turbulence, coherent structures, dynamical systems and symmetry, Cambridge monograph on mechanics, 1996

[26] E. Gaudard, Ph. Druault, R. Marchiano, F. Van Herpe, About the pod application for separating acoustic and turbulent fluctuations from wall pressure synthesised field, Int. J. Aerodynamics 4 (2014) 108-133

[27] S. Lu, P. Sagaut, Pseudo-characteristic formulation and dynamic boundary conditions for computational aeroacoustics, Int. J. Num. Meth. Fluids 53 (2007) 201-227

[28] A. Deneuve, Ph. Druault, R. Marchiano, P. Sagaut, A coupled time-reversal complex differentiation method for aeroacoustic sensitivity analysis: towards a source detection procedure, J. Fluid Mech. 642 (2010) 181-212

[29] Ph. Druault, R. Marchiano, P. Sagaut, Localization of aeroacoustics sound sources in viscous flows by a time reversal method, J. Sound Vib. 332 (2013) 3655-3669

[30] J. Sesterhenn, A characteristic-type formulation of the Navier-Stokes equations for high order upwind schemes, Comput. Fluids 30 (2001) 37-67

[31] OpenFOAM: The Open Source CFD Toolbox, http://foam. sourceforge.net/docs/Guides-a4/ ProgrammersGuide.pdf (2014). 
[32] H. Weller, G. Tabor, H. Jasak, C. Fureby, A tensorial approach to computational continuum mechanics using object-oriented techniques, Comput. Phys. 12 (1998) 620-631

[33] E. Gaudard, Développement d'outils pour l'analyse de champs aéroacoustiques à l'origine du bruit dans un habitacle automobile, Ph.D. thesis, University Pierre et Marie Curie, Paris 6, 2014

[34] N.M. Newmark, A method of computation for structural dynamics, J. Eng. Mech. Division (Proceedings of the ASCE) (1959) 67-94

[35] M. Ji, M. Wang, Surface pressure fluctuations on steps immersed in turbulent boundary layers, J. Fluid Mech. 712 (2012) 471-504

[36] Y. Moon, Y. Bae, M.H. Cho, Numerical investigation of the aerodynamic noise from a forward facing step, in: ECCOMAS CFD Conference, 2006

[37] S. Roudnitzky, Ph. Druault, P. Guibert, Proper Orthogonal Decomposition of in-cylinder engine flow into mean component, coherent structures and random Gaussian fluctuations, J. Turbulence 7 (2006) 1-19
[38] P. Druault, C. Chaillou, Use of Proper Orthogonal Decomposition for reconstructing the $3 \mathrm{D}$ in-cylinder mean-flow field from PIV data, C.R. Mecanique 335 (2007) 42-47

[39] J. Lumley, The structure of inhomogeneous turbulent flows, in: Yaglom, Tatarsky (Eds.), Atm. Turb. and Radio wave Prop., 1967, pp. 166-178

[40] A. Hekmati, D. Ricot, Ph. Druault, About the convergence of POD and EPOD modes computed from CFD simulation, Comput. Fluids 50 (2011) 60-71

[41] S. Ravindran, Control of flow separation over a forwardfacing step by model reduction, Comput. Methods Appl. Mech. Eng. 191 (2002) 4599-4617

[42] J.W. Cooley, J.W. Tukey, An algorithm for the machine calculation of complex fourier series, Math. Comput. 19 (1965) 297-301 